\title{
Type- 1 and Type-2 effective Takagi-Sugeno fuzzy models for decentralized control of multi-input-multi-output processes
}

\author{
Qian-Fang Liao ${ }^{a}$, Da Sun ${ }^{\mathrm{b}}$, Wen-Jian Cai ${ }^{\mathrm{a}, *}$, Shao-Yuan $\mathrm{Li}^{\mathrm{c}}$, You-Yi Wang ${ }^{\mathrm{a}}$ \\ a School of Electrical and Electronic Engineering, Nanyang Technological University, 639798, Singapore \\ ${ }^{\mathrm{b}}$ Department of Biomedical Engineering, National University of Singapore, 118633, Singapore

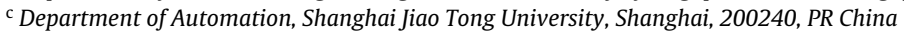

\section{A R T I C L E I N F O}

\section{Article history:}

Received 5 November 2015

Received in revised form 13 January 2017

Accepted 16 January 2017

Available online 16 February 2017

\section{Keywords:}

Interactions

Loop pairing

Effective Takagi-Sugeno (T-S) fuzzy model

Type-2 fuzzy system

Decentralized control

\begin{abstract}
A B S T R A C T
Effective model is a novel tool for decentralized controller design to handle the interconnected interactions in a multi-input-multi-output (MIMO) process. In this paper, Type- 1 and Type-2 effective Takagi-Sugeno fuzzy models (ETSM) are investigated. By means of the loop pairing criterion, simple calculations are given to build Type-1/Type-2 ETSMs which are used to describe a group of non-interacting equivalent single-input-single-output (SISO) systems to represent an MIMO process, consequently the decentralized controller design can be converted to multiple independent single-loop controller designs, and enjoy the well-developed linear control algorithms. The main contributions of this paper are: i) Compared to the existing T-S fuzzy model based decentralized control methods using extra terms to characterize interactions, ETSM is a simple feasible alternative; ii) Compared to the existing effective model methods using linear transfer functions, ETSM can be carried out without requiring exact mathematical process functions, and lays a basis to develop robust controllers since fuzzy system is powerful to handle uncertainties; iii) Type-1 and Type-2 ETSMs are presented under a unified framework to provide objective comparisons. A nonlinear MIMO process is used to demonstrate the ETSMs' superiority over the effective transfer function (ETF) counterparts as well as the evident advantage of Type-2 ETSMs in terms of robustness. A multi-evaporator refrigeration system is employed to validate the practicability of the proposed methods.
\end{abstract}

() 2017 Elsevier Ltd. All rights reserved.

\section{Introduction}

In the area of multi-input-multi-output (MIMO) process control, the Takagi-Sugeno (T-S) fuzzy model based decentralized control is an attractive topic because of its outstanding merits including: i). it is easy to design and tune because it uses the simplest control structure where each manipulated variable (process input) is determined by only one controlled variable (process output); ii) no exact mathematical process functions are required since fuzzy models can be built to a high degree of accuracy from data samples and expert experience [1,2]; iii) it is robust to disturbance since fuzzy system excels in handling uncertainties [1-3]; iv) linear control algorithms can be applied to design controllers for a nonlinear process via parallel distributed compensation [4] since the T-S fuzzy model is composed of a group of linear local models [3,4]. A number of academic results concerning this topic have been proposed. Such as the networked and robust decentralized control for large-scale and interconnected MIMO processes in [5-8]. The main difficulty for decentralized control is to deal with the interactions among the paired input-output control-loops due to its limited control structure flexibility. In the existing T-S fuzzy model based methods, generally, for a certain control pair, extra terms are added to its individual open-loop model to characterize the interacting effects from other loops. A simple example is given as follows:

$$
\begin{array}{rrr}
\text { Rule } & l: I F & u_{j} \text { is } C^{l} \\
& \text { THEN } \quad y_{i}=a_{i j}^{l} \cdot u_{j}+\sum_{k=1, k \neq j}^{n} \xi_{k}\left(u_{k}\right), \quad l=1, \cdots, M
\end{array}
$$

\footnotetext{
* Corresponding author.

E-mail address: ewjcai@ntu.edu.sg (W.-J. Cai).
} 
where $M$ is the number of fuzzy rules; $y_{i}$ is the $i$ th output and $u_{j}$ is the $j$ th input $(i, j=1, \ldots, n)$, and $y_{i}-u_{j}$ is one of the control pairs of an $n \times n$ process; $C^{l}$ is a fuzzy set; $y_{i}=a_{i j}^{l} \cdot u_{j}$ is the $l$ th local model of the T-S fuzzy model for the individual open-loop $y_{i}-u_{j}$ and $a_{i j}^{l}$ is the coefficient; $\xi_{k}\left(u_{k}\right)$ is an extra term standing for the interactions caused by $u_{k}$, and $\sum_{k=1, k \neq j}^{n} \xi_{k}\left(u_{k}\right)$ is the sum of extra terms to describe the total interacting effects. Each local controller of a decentralized control system is devised based on the model of a control pair bearing extra terms as shown in Eq. (1) to cope with interactions. However, several problems may arise:

- For a large-scale process, a large number of extra terms need to be identified, which would drastically increase the cost in process modeling;

- For a complex process, the interactions may not be directly measured or evaluated, which would form obstacles to deriving the extra terms;

- For a nonlinear process, different working conditions may require different control pair configurations and result in changed coupling effects, which would lead to challenges in finding suitable extra terms to describe the varying interactions;

- The local models of a T-S fuzzy model may not be linear after adding the extra terms, which would increase the complexity for controller design.

Given the above problems, a more practical method to express the interactions is required. One interesting manner developed recently is to create the effective models. For each control pair, an effective model can be built by revising the coefficients of its individual open-loop model to reflect the interacting effects. Using the example in Eq. (1), a simple effective T-S fuzzy model (ETSM) can be expressed as:

$$
\begin{aligned}
& \text { Rule } l: I F \quad u_{j} \text { is } C^{l} \\
& \text { THEN } y_{i}=\hat{a}_{i j}^{l} \cdot u_{j}, \quad l=1, \cdots, M
\end{aligned}
$$

where $\hat{a}_{i j}^{l}$ is the revised coefficient. Compared to Eq. (1), ETSM in Eq. (2) uses a different manner to express interactions that can solve the aforementioned problems caused by using extra terms, and greatly simplify decentralized controller design because: i) the ETSM method is using a group of non-interacting single-input single-output (SISO) systems to represent an MIMO process such that the decentralized controller design can be decomposed into multiple independent single-loop controller designs; ii) the ETSM retains the linearity in each of its local models which provides a platform to apply the mature linear methods to regulate a nonlinear process. How to revise the coefficients to achieve an ETSM that can correctly reflect the interacting effects is a key problem to solve. Currently several methods taking advantage of loop pairing criteria to construct effective models are available. A loop pairing criterion is used to pair inputs and outputs to determine a proper decentralized control structure with minimum coupling effects among the paired control-loops, and it provides quantified interconnected interactions to calculate the revised coefficients in effective models. In [9], an approach was presented to derive effective transfer functions (ETF) to describe a group of equivalent open-loop processes for decentralized control in terms of dynamic relative gain array (RGA) [10-13] based criterion, and [14] proposed a model reduction technique to simplify the effective open-loop transfer function of [9]. In [15], the method to build ETFs using effective relative gain array [16] based criterion was introduced. In [17], an algorithm to modify the coefficients for ETF construction according to relative normalized gain array (RNGA) [18] based criterion was developed. The simulations or experiments in $[9,14,17,18]$ demonstrated the better performances of ETF based control methods when compared to several other popular control tuning approaches. Among these, RNGA based effective model has prominent advantages that it provides a comprehensive description of dynamic interactions, and works with satisfactory performances for both low and high dimensional processes and without requiring the specifics of controllers, and is able to provide a unique result with less computational complexity [17,18]. We investigated RNGA based ETSM for decentralized control in a conference article [19], which is, to the best of author's knowledge, the first work in the area of loop pairing criterion based effective fuzzy model. Compared to the existing ETF methods, ETSM is an alternative to process controller design where exact mathematical functions are unavailable. Moreover, it lays a basis to develop robust controller since fuzzy system is strong in compensating for uncertainties.

The ETSM studied in [19] is based on traditional (Type-1) fuzzy models where the fuzzy memberships are crisp numbers. When large uncertainties appear, the crisp fuzzy memberships may struggle to describe the conditions. In this case, Type-2 fuzzy model [20-22] with the fuzzy memberships that are themselves fuzzy can be applied. In a Type-2 fuzzy set, the fuzzy membership of an element includes primary and secondary grades that can be considered as a Type-1 fuzzy set. As shown in Fig. 1, Part (a) is a general Type-2 fuzzy set where the secondary grades range from 0 to 1 . When all secondary grades are either 0 or 1 that the fuzzy membership for an element is an interval, it becomes an interval Type-2 fuzzy set as shown in Part (b) which is more widely used because of its manageable calculations [23]. The increased fuzziness endows a Type-2 fuzzy set additional design degrees of freedom that make it possible to directly describe the uncertainties [20-23]. [24] gave an introduction of Type-2 T-S fuzzy models, and several results [25-27] proved that Type-2 T-S fuzzy model outperforms its Type- 1 counterpart in terms of accuracy and robustness in process modeling and control.

This paper investigates both Type- 1 and Type-2 ETSM for decentralized control. Firstly, the identification of Type- 1 and Type- 2 T-S fuzzy models for an MIMO process based on data samples is given. Afterwards, by means of RNGA based criterion, the input-output pairing configuration is determined and simple calculations are introduced to construct Type- 1 and Type-2 ETSMs. A numerical nonlinear MIMO process is used to demonstrate the superiorities of ETSMs over their ETF counterparts, as well as the evident advantage of Type-2 ETSMs with respect to robustness. An experimental refrigeration system is used to validate the practicability of the proposed methods and compare Type- 1 and Type-2 ETSMs in a real application. The main contributions of this work are:

i) Compared to the existing T-S fuzzy model based decentralized control methods using extra terms to characterize interactions, ETSM method expresses the interacting effects through revising the coefficients of the original T-S fuzzy model, which is a simple feasible alternative;

ii) Compared to the existing ETF methods, ETSM does not require accurate mathematical process functions, and lays a basis to develop robust controllers since fuzzy system is a powerful tool to handle uncertainties; 


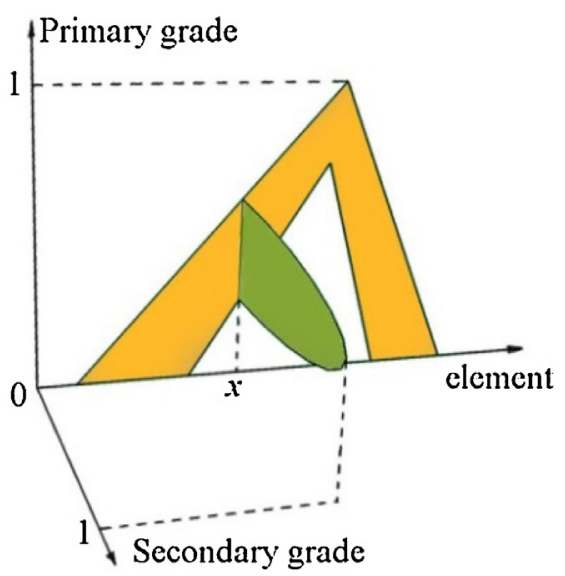

(a)

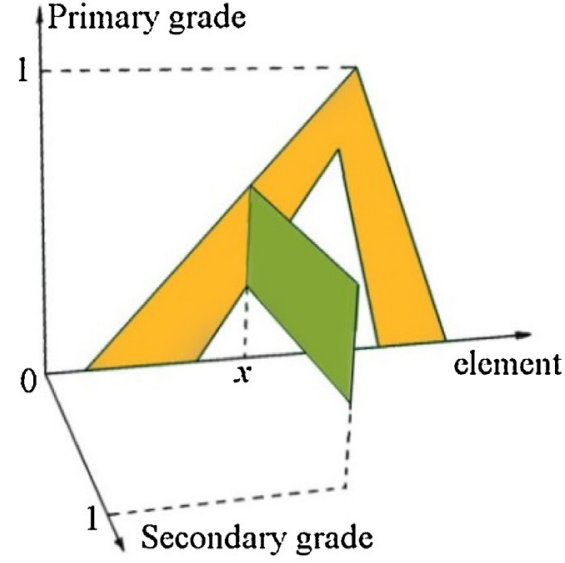

(b)

Fig 1. (a) General Type-2 fuzzy set, secondary grades are in [0,1] (b) Interval Type-2 fuzzy set, secondary grades are 0 or 1 .

iii) Type-2 ETSM is proposed to enrich the ETSM study and offers an improvement in terms of robustness. Also, Type-1 and Type-2 ETSM are presented under a unified framework to allow objective comparisons.

\section{T-S fuzzy modeling for an MIMO process}

Throughout this paper, it is assumed that the MIMO processes considered are open-loop stable, nonsingular at the steady-state conditions, and square in dimension (equal number of inputs and outputs). The following T-S fuzzy model matrix can be used to describe an MIMO process with $n$ outputs $\left(y_{i}, i=1, \ldots, n\right)$ and $n$ inputs $\left(u_{j}, j=1, \ldots, n\right)[19,28]$ :

$$
\boldsymbol{F}_{T S}=\left[f_{T S, i j}\right]_{n \times n}=\left[\begin{array}{cccc}
f_{T S, 11} & f_{T S, 12} & \cdots & f_{T S, 1 n} \\
f_{T S, 21} & f_{T S, 22} & \cdots & f_{T S, 2 n} \\
\vdots & \vdots & \ddots & \vdots \\
f_{T S, n 1} & f_{T S, n 2} & \cdots & f_{T S, n n}
\end{array}\right]
$$

where $f_{T S, i j}$ is the individual open-loop T-S fuzzy model for $y_{i}-u_{j}$, which is always identifiable through proper excitations [29]. When $f_{T S, i j}$ is a Type-1 fuzzy model, its fuzzy rules can be expressed as:

$$
\begin{array}{rc}
\text { Rule } l: \text { IF } & \boldsymbol{x}_{i j}(k) \text { is } \quad C_{i j}^{l} \\
\text { THEN } & y_{i}^{l}(k)=a_{i j, 0}^{l} \cdot u_{j}\left(k-\tau_{i j}\right)+a_{i j, 1}^{l} \cdot u_{j}\left(k-\tau_{i j}-1\right)+\cdots+a_{i j, p}^{l} \cdot u_{j}\left(k-\tau_{i j}-p\right) \\
& +b_{i j, 1}^{l} \cdot y_{i}(k-1)+\cdots+b_{i j, q}^{l} \cdot y_{i}(k-q)
\end{array}
$$

where $l=1, \ldots, M_{i j}, M_{i j}$ is the number of fuzzy rules in $f_{T S, i j} . \boldsymbol{x}_{i j}(k) \in \mathbb{R}^{n}$ is a vector consisting of past inputs and outputs as: $\boldsymbol{x}_{i j}(k)=$ $\left[\begin{array}{lllllll}u_{j}\left(k-\tau_{i j}\right) & \cdots & u_{j}\left(k-\tau_{i j}-p\right) & y_{i}(k-1) & \cdots & y_{i}(k-q)\end{array}\right]^{T}, p$ and $q$ are integers, $\tau_{i j}=\tau_{i j}^{\prime} / T, \tau_{i j}^{\prime}$ denotes the time delay in $y_{i}-u_{j}$, and $T$ is the sampling interval; $y_{i}^{l}(k)$ is the output of $l$ th fuzzy rule; $a_{i j, r}^{l}(r=0,1, \ldots, p)$ and $b_{i j, s}^{l}(s=1, \ldots, q)$ are the coefficients. The output of $f_{T S, i j}$ is a weighted sum of local outputs:

$$
y_{i}(k)=\sum_{l=1}^{M_{i j}} \mu_{i j}^{l}\left(\boldsymbol{x}_{i j}(k)\right) y_{i}^{l}(k)
$$

$\mu_{i j}^{l}\left(\boldsymbol{x}_{i j}(k)\right)$ denotes the fuzzy membership function of $\boldsymbol{x}_{i j}(k)$ in the $l$ th fuzzy set $C_{i j}^{l}$. As the weights, they satisfy $0 \leq \mu_{i j}^{l}\left(\boldsymbol{x}_{i j}(k)\right) \leq 1$ and $\sum_{l=1}^{M_{i j}} \mu_{i j}^{l}\left(\boldsymbol{x}_{i j}(k)\right)=1$.

When $f_{T S, i j}$ is an interval Type-2 T-S fuzzy model, its fuzzy rules can be expressed as:

$$
\begin{aligned}
\text { Rule } l: \text { IF } & \boldsymbol{x}_{i j}(k) \text { is } \quad \tilde{C}_{i j}^{l} \\
\text { THEN } & \tilde{y}_{i}^{l}(k)=\tilde{a}_{i j, 0}^{l} \cdot u_{j}\left(k-\tau_{i j}\right)+\tilde{a}_{i j, 1}^{l} \cdot u_{j}\left(k-\tau_{i j}-1\right)+\cdots+\tilde{a}_{i j, p}^{l} \cdot u_{j}\left(k-\tau_{i j}-p\right) \\
& +\tilde{b}_{i j, 1}^{l} \cdot y_{i}(k-1)+\cdots+\tilde{b}_{i j, q}^{l} \cdot y_{i}(k-q)
\end{aligned}
$$

$l=1, \ldots, M_{i j}$, where $\tilde{C}_{i j}^{l}$ is an interval Type-2 fuzzy set. The fuzzy membership of $\boldsymbol{x}_{i j}(k)$ in $\tilde{C}_{i j}^{l}$ is an interval denoted as $\tilde{\mu}_{i j}^{l}\left(\boldsymbol{x}_{i j}(k)\right)=$ $\left[\mu_{i j, l b}^{l}\left(\boldsymbol{x}_{i j}(k)\right), \quad \mu_{i j, r b}^{l}\left(\boldsymbol{x}_{i j}(k)\right)\right]$ where $\mu_{i j, l b}^{l}\left(\boldsymbol{x}_{i j}(k)\right)$ and $\mu_{i j, r b}^{l}\left(\boldsymbol{x}_{i j}(k)\right)$ are left and right bounds respectively; The local model's coefficients 
are also intervals as $\tilde{a}_{i j, r}^{l}=\left[a_{i j, l b, r}^{l}, \quad a_{i j, r b, r}^{l}\right](r=0,1, \ldots, p)$ and $\tilde{b}_{i j, s}^{l}=\left[b_{i j, l b, s}^{l}, \quad b_{i j, r b, s}^{l}\right](s=1, \ldots, q)$, and the output of $l$ th fuzzy rule is $\tilde{y}_{i}^{l}(k)=\left[y_{i, l b}^{l}(k), \quad y_{i, r b}^{l}(k)\right]$ that can be obtained by [24]:

$$
\left\{\begin{array}{l}
y_{i, l b}^{l}(k)=a_{i j, l b, 0}^{l} \cdot u_{j}\left(k-\tau_{i j}\right)+\cdots+a_{i j, l b, p}^{l} \cdot u_{j}\left(k-\tau_{i j}-p\right)+b_{i j, l b, 1}^{l} \cdot y_{i}(k-1)+\cdots+b_{i j, l b, q}^{l} \cdot y_{i}(k-q) \\
y_{i, r b}^{l}(k)=a_{i j, r b, 0}^{l} \cdot u_{j}\left(k-\tau_{i j}\right)+\cdots+a_{i j, r b, p}^{l} \cdot u_{j}\left(k-\tau_{i j}-p\right)+b_{i j, r b, 1}^{l} \cdot y_{i}(k-1)+\cdots+b_{i j, r b, q}^{l} \cdot y_{i}(k-q)
\end{array}\right.
$$

Based on $M_{i j}$ fuzzy rules, a type-reduced set [24], denoted by $\tilde{y}_{i}(k)$ can be derived:

$$
\tilde{y}_{i}(k)=\left[y_{i, l b}(k), \quad y_{i, r b}(k)\right]
$$

where $y_{i, l b}(k)$ and $y_{i, r b}(k)$ can be calculated by Karnik-Mendel method [24]. However, Karnik-Mendel method requires iterative calculations that may be time consuming. In this paper, the following calculations $[25,27]$ is selected for simplification:

$$
\left\{\begin{array}{l}
y_{i, l b}(k)=\sum_{l=1}^{M_{i j}} \mu_{i j, l b}^{l}\left(\boldsymbol{x}_{i j}(k)\right) \cdot y_{i, l b}^{l}(k) / \sum_{l=1}^{M_{i j}} \mu_{i j, l b}^{l}\left(\boldsymbol{x}_{i j}(k)\right) \\
y_{i, r b}(k)=\sum_{l=1}^{M_{i j}} \mu_{i j, r b}^{l}\left(\boldsymbol{x}_{i j}(k)\right) \cdot y_{i, r b}^{l}(k) / \sum_{l=1}^{M_{i j}} \mu_{i j, r b}^{l}\left(\boldsymbol{x}_{i j}(k)\right)
\end{array}\right.
$$

Note that in an Type-2 fuzzy set, $\sum_{l=1}^{M_{i j}} \mu_{i j, l b}^{l}\left(\boldsymbol{x}_{i j}(k)\right)$ and $\sum_{l=1}^{M_{i j}} \mu_{i j, r b}^{l}\left(\boldsymbol{x}_{i j}(k)\right)$ may not be equal to 1 . The crisp output can be obtained by defuzzifying $\tilde{y}_{i}(k)$ as $[25,27]$ :

$$
y_{i}(k)=\frac{y_{i, l b}(k)+y_{i, r b}(k)}{2}
$$

Both Type-1 and Type-2 T-S fuzzy model can be constructed based on the input-output data samples that are briefly introduced as follows [25]:

i) For an input-output channel $y_{i}-u_{j}$, collect $N_{i j}$ data samples as $\boldsymbol{z}_{i j}(k)=\left[\boldsymbol{x}_{i j}(k)^{T} \quad y_{i}(k)\right]^{T}, k=1, \ldots, N_{i j}$. Determine the number of fuzzy rules $M_{i j}$, which implies $M_{i j}$ fuzzy sets/clusters will be used to characterize the data.

ii) Use Gustafson-Kessel clustering algorithm [30] to locate $M_{i j}$ fuzzy cluster centers $\boldsymbol{z}_{c, i j}^{l}=\left[\left(\boldsymbol{x}_{c, i j}^{l}\right)^{T} \quad y_{c, i}^{l}\right]^{T}\left(l=1, \ldots . M_{i j}\right)$, where $\boldsymbol{x}_{c, i j}^{l}=$ $\left[\begin{array}{lll}u_{c, j^{-}}^{l} \tau_{i j} & y_{c, i^{-}}^{l} 1 & y_{c, i}^{l}{ }^{2}\end{array}\right]^{T}$ is the $l$ th center of input vectors. Denote the distance between $\boldsymbol{z}_{i j}(k)$ and $\boldsymbol{z}_{c, i j}^{l}$ as $D_{i j}^{l}\left(\boldsymbol{z}_{i j}(k)\right)=\left(\boldsymbol{z}_{i j}(k)-\boldsymbol{z}_{c, i j}^{l}\right)^{T} \cdot \boldsymbol{A}_{i j}$. $\left(\boldsymbol{z}_{i j}(k)-\boldsymbol{z}_{c, i j}^{l}\right)\left(l=1, \ldots M_{i j}\right)$, where $\boldsymbol{A}_{i j}$ is the norm-inducing matrix calculated based on data samples. $D_{i j}^{l}\left(\boldsymbol{z}_{i j}(k)\right)$ 's $\left(l=1, \ldots M_{i j}\right)$ determine the Type- 1 fuzzy memberships for $\boldsymbol{z}_{i j}(k)$ as:

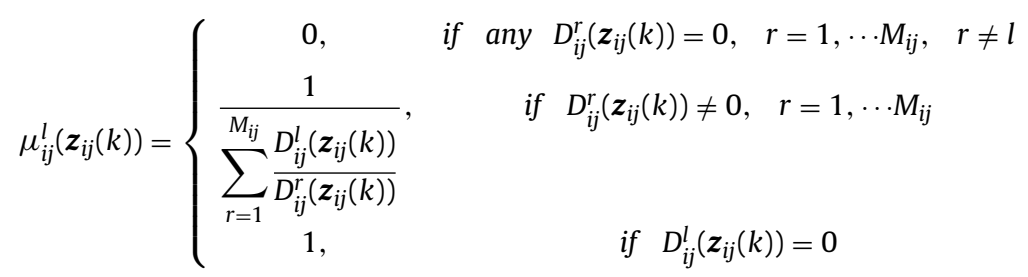

iii) Assign each datum to the cluster where it has the largest Type-1 fuzzy membership to divide the data into $M_{i j}$ groups. For each group, utilize least square method to identify the coefficients $a_{i j, r}^{l}(r=0,1, \ldots, p)$ and $b_{i j, s}^{l}(s=1, \ldots, q)$ for its associated Type-1 fuzzy rule.

iv) In each group, evaluate a variant range for fuzzy membership, $\Delta \mu_{i j}^{l}>0$, to achieve an interval Type-2 fuzzy membership $\tilde{\mu}_{i j}^{l}\left(\boldsymbol{z}_{i j}(k)\right)=$ $\left[\mu_{i j, l b}^{l}\left(\boldsymbol{z}_{i j}(k)\right), \quad \mu_{i j, r b}^{l}\left(\boldsymbol{z}_{i j}(k)\right)\right]$ for each datum $\boldsymbol{z}_{i j}(k)$ based on its Type-1 fuzzy membership as:

$$
\left\{\begin{array}{l}
\mu_{i j, l b}^{l}\left(\boldsymbol{z}_{i j}(k)\right)=\max \left\{0, \quad \mu_{i j}^{l}\left(\boldsymbol{z}_{i j}(k)\right)-\Delta \mu_{i j}^{l}\right\} \\
\mu_{i j, r b}^{l}\left(\boldsymbol{z}_{i j}(k)\right)=\min \left\{\mu_{i j}^{l}\left(\boldsymbol{z}_{i j}(k)\right)+\Delta \mu_{i j}^{l}, \quad 1\right\}
\end{array}\right.
$$

v) In each group, evaluate a variant range for output, $\Delta y_{i}>0$, such that two data, denoted as $\boldsymbol{z}_{i j, l b}(k)$ and $\boldsymbol{z}_{i j, r b}(k)$, can be derived from each datum $z_{i j}(k)$ as

$$
\begin{cases}\boldsymbol{z}_{i j, l b}(k)=\left[\begin{array}{ll}
\boldsymbol{x}_{i j}(k)^{T} & y_{i}(k)-\Delta y_{i}
\end{array}\right]^{T}=\left[\begin{array}{ll}
\boldsymbol{x}_{i j}(k)^{T} & y_{i, l b}(k)
\end{array}\right]^{T} \\
\boldsymbol{z}_{i j, r b}(k)=\left[\begin{array}{ll}
\boldsymbol{x}_{i j}(k)^{T} & y_{i}(k)+\Delta y_{i}
\end{array}\right]^{T}=\left[\begin{array}{ll}
\boldsymbol{x}_{i j}(k)^{T} & y_{i, r b}(k)
\end{array}\right]^{T}\end{cases}
$$

Use least square method to identify the coefficients of two linear polynomials as in Eq. (7) based on $\boldsymbol{z}_{i j, l b}(k)$ and $\boldsymbol{z}_{i j, r b}(k)$ respectively to have the left and right bounds of $\tilde{a}_{i j, r}^{l}(r=0,1, \ldots, p)$ and $\tilde{b}_{i j, s}^{l}(s=1, \ldots, q)$, for its associated Type-2 fuzzy rule.

When given a new input $\boldsymbol{x}_{i j}(k)$, its Type- 1 fuzzy memberships $\mu_{i j}^{l}\left(\boldsymbol{x}_{i j}(k)\right), l=1, \ldots, M_{i j}$, are calculated by:

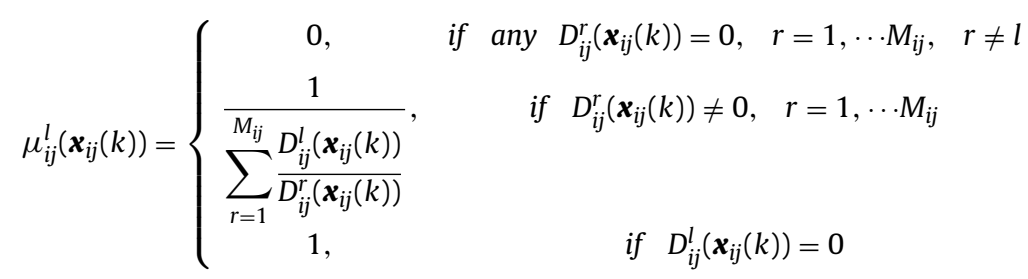




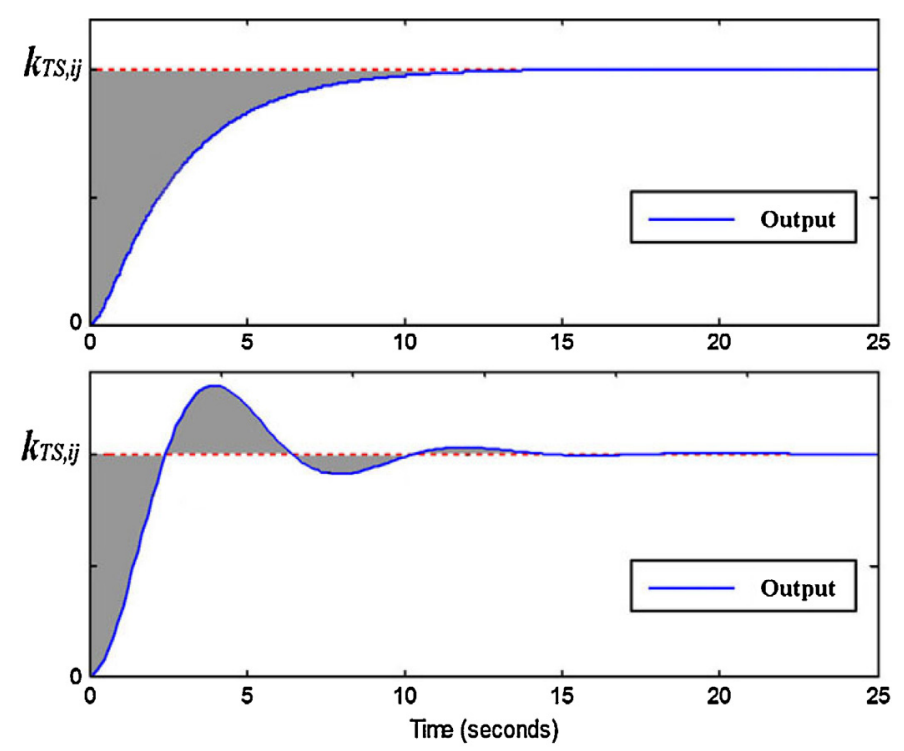

Fig. 2. Two typical unit step responses.

where $D_{i j}^{l}\left(\boldsymbol{x}_{i j}(k)\right)=\left(\boldsymbol{x}_{i j}(k)-\boldsymbol{x}_{c, i j}^{l}\right)^{T} \cdot\left(\boldsymbol{x}_{i j}(k)-\boldsymbol{x}_{c, i j}^{l}\right)$. The output from the Type-1 T-S fuzzy model is calculated by Eq. (5). While its Type-2 fuzzy memberships $\tilde{\mu}_{i j}^{l}\left(\boldsymbol{x}_{i j}(k)\right)=\left[\mu_{i j, l b}^{l}\left(\boldsymbol{x}_{i j}(k)\right), \quad \mu_{i j, r b}^{l}\left(\boldsymbol{x}_{i j}(k)\right)\right]$ are:

$$
\left\{\begin{array}{l}
\mu_{i j, l b}^{l}\left(\boldsymbol{x}_{i j}(k)\right)=\max \left\{\mu_{i j}^{l}\left(\boldsymbol{x}_{i j}(k)\right)-\Delta \mu_{i j}^{l}, \quad 0\right\} \\
\mu_{i j, r b}^{l}\left(\boldsymbol{x}_{i j}(k)\right)=\min \left\{\mu_{i j}^{l}\left(\boldsymbol{x}_{i j}(k)\right)+\Delta \mu_{i j}^{l}, \quad 1\right\}
\end{array}\right.
$$

The output from the Type-2 T-S fuzzy model is calculated by Eqs. (9) and (10).

\section{Relative normalized gain array based loop pairing criterion}

Loop pairing defines the decentralized control-loop configuration, i.e., which of the available inputs should be chosen to manipulate each of the process outputs. From a T-S fuzzy model, two factors can be calculated for interaction assessment according to RNGA based criterion [18,28]: steady-state gain, $k_{T S, i j}$, which indicates the effect of $u_{j}$ on the gain of $y_{i}$ when the process reaches the steady-state condition, and normalized integrated error, $e_{T S, i j}$, which reflects the response speed of $y_{i}$ to $u_{j}$. Both $k_{T S, i j}$ and $e_{T S, i j}$ are defined from the step response. Two examples are given in Fig. 2, where the shaded area and $k_{T S, i j}$ determine $e_{T S, i j}$ as [28]:

$$
e_{T S, i j}=\sum_{r=0}^{\infty} \frac{y_{i}(\infty)-y_{i}(r \cdot T)}{k_{T S, i j}} \cdot T
$$

where $T$ is the sampling interval, $y_{i}(\infty)=\left.y_{i}(k)\right|_{k \rightarrow \infty}$ is the steady-state output of $f_{T S, i j}$ in unit step response. It is easy to know that $y_{i}(\infty)=k_{T S, i j}$. $y_{i}(r \cdot T)$ is the output at $r$ th sampling time. $e_{T S, i j}$ can be used to represent the dynamic property since smaller/larger $e_{T S, i j}$ means $y_{i}$ gives faster/slower response to $u_{j}[18,28]$.

Because of the nonlinear nature in a fuzzy model, an operating point should be given to calculate $k_{T S, i j}$ and $e_{T S, i j}$ from $f_{T S, i j} \operatorname{since}$ different operating conditions may have different $k_{T S, i j}$ and $e_{T S, i j}$ and result in different control configurations [28]. Given an operating point for $f_{T S, i j}$ as:

$$
\boldsymbol{x}_{0, i j}=\left[\begin{array}{llllll}
u_{0, j}\left(k_{0}-\tau_{i j}\right) & \cdots & u_{0, j}\left(k_{0}-\tau_{i j}-p\right) & y_{0, i}\left(k_{0}-1\right) & \cdots & y_{0, i}\left(k_{0}-q\right)
\end{array}\right]^{T}
$$

In the vicinity of $\boldsymbol{x}_{0, i j}$, a T-S fuzzy model can be approximately represented by a linear function by letting $\mu_{i j}^{l}\left(\boldsymbol{x}_{i j}(k)\right)=\mu_{i j}^{l}\left(\boldsymbol{x}_{0, i j}\right)[28]$ :

$$
\begin{aligned}
& y_{i}(k)=f_{T S, i j}\left(\boldsymbol{x}_{i j}(k)\right) \\
& =a_{i j, 0} \cdot u_{j}\left(k-\tau_{i j}\right)+\cdots+a_{i j, p} \cdot u_{j}\left(k-\tau_{i j}-p\right)+b_{i j, 1} \cdot y_{i}(k-1)+\cdots+b_{i j, q} \cdot y_{i}(k-q)
\end{aligned}
$$

When $f_{T S, i j}$ is a Type-1 T-S fuzzy model as in Eq. (4), the coefficients of Eq. (18) are

$$
\begin{cases}a_{i j, r}=\sum_{l=1}^{M_{i j}} \mu_{i j}^{l}\left(\boldsymbol{x}_{0, i j}\right) a_{i j, r}^{l}, & r=0,1, \cdots, p \\ b_{i j, s}=\sum_{l=1}^{M_{i j}} \mu_{i j}^{l}\left(\boldsymbol{x}_{0, i j}\right) b_{i j, s}^{l}, & s=1, \cdots, q\end{cases}
$$

When $f_{T S, i j}$ is a Type-2 T-S fuzzy model as in Eq. (6), the coefficients of Eq. (18) are

$$
\begin{cases}a_{i j, r}=\left(a_{i j, l b, r}+a_{i j, r b, r}\right) / 2, & r=0,1, \cdots, p \\ b_{i j, s}=\left(b_{i j, l b, s}+b_{i j, r b, s}\right) / 2, & s=1, \cdots, q\end{cases}
$$


where $\quad a_{i j, l b, r}=\sum_{l=1}^{M_{i j}} \mu_{i j, l b}^{l}\left(\boldsymbol{x}_{0, i j}\right) \cdot a_{i j, l b, r}^{l} / \sum_{l=1}^{M_{i j}} \mu_{i j, l b}^{l}\left(\boldsymbol{x}_{0, i j}\right), \quad a_{i j, r b, r}=\sum_{l=1}^{M_{i j}} \mu_{i j, r b}^{l}\left(\boldsymbol{x}_{0, i j}\right) \cdot a_{i j, r b, r}^{l} / \sum_{l=1}^{M_{i j}} \mu_{i j, r b}^{l}\left(\boldsymbol{x}_{0, i j}\right), \quad b_{i j, l b, s}=\sum_{l=1}^{M_{i j}} \mu_{i j, l b}^{l}\left(\boldsymbol{x}_{0, i j}\right)$. $b_{i j, l b, s}^{l} / \sum_{l=1}^{M_{i j}} \mu_{i j, l b}^{l}\left(\boldsymbol{x}_{0, i j}\right)$ and $b_{i j, r b, s}=\sum_{l=1}^{M_{i j}} \mu_{i j, r b}^{l}\left(\boldsymbol{x}_{0, i j}\right) \cdot b_{i j, r b, s}^{l} / \sum_{l=1}^{M_{i j}} \mu_{i j, r b}^{l}\left(\boldsymbol{x}_{0, i j}\right)$. Based on Eq. (18), $k_{T S, i j}$ and $e_{T S, i j}$ can be calculated by following equations [28]:

$$
\begin{aligned}
k_{T S, i j}= & \frac{a_{i j, 0}+a_{i j, 1}+\cdots+a_{i j, p}}{1-\left(b_{i j, 1}+b_{i j, 2}+\cdots+b_{i j, q}\right)} \\
e_{T S, i j} & =\frac{\sum_{r=0}^{p} r a_{i j, r}-\sum_{w=0}^{p} \sum_{s=1}^{q} a_{i j, w} \cdot b_{i j, s} \cdot|w-s| \cdot \operatorname{sgn}(w-s)}{\left(a_{i j, 0}+a_{i j, 1}+\cdots+a_{i j, p}\right)\left(1-\left(b_{i j, 1}+\cdots+b_{i j, q}\right)\right)} \cdot T+\tau_{i j} \cdot T
\end{aligned}
$$

Eqs. (21) and (22) could be very simple for real applications since $p$ and $q$ are generally not large. For example, when $p=0$ and $q=2$, they become:

$$
k_{T S, i j}=\frac{a_{i j, 0}}{1-\left(b_{i j, 1}+b_{i j, 2}\right)}, e_{T S, i j}=\frac{b_{i j, 1}+2 b_{i j, 2}}{1-\left(b_{i j, 1}+b_{i j, 2}\right)} \cdot T+\tau_{i j} \cdot T
$$

Collecting the calculated results of Eqs. (21) and (22) of each element in $\boldsymbol{F}_{T S}$ forms a steady-state gain matrix $\boldsymbol{K}_{T S}=\left[k_{T S, i j}\right]_{n \times n}$ and a normalized integrated error matrix $\boldsymbol{E}_{T S}=\left[e_{T S, i j}\right]_{n \times n}$. Next, we introduce the important concepts of RNGA loop pairing criterion as follows:

RGA: the relative gain of a control pair $y_{i}-u_{j}$, denoted by $\lambda_{T S, i j}$, is defined as [10]:

$$
\lambda_{T S, i j}=\frac{k_{T S, i j}}{\hat{k}_{T S, i j}}
$$

where $\hat{k}_{T S, i j}$ is the steady-state gain of $y_{i}-u_{j}$ when all other control-loops are closed. RGA is an array formed by assembling all the relative gains as RGA $=\left[\lambda_{T S, i j}\right]_{n \times n}$, which can be calculated only using individual open-loop information [12]:

$$
\mathrm{RGA}=\boldsymbol{K}_{T S} \otimes \boldsymbol{K}_{T S}^{-T}
$$

where $\otimes$ is element-by-element product, $\boldsymbol{K}_{T S}^{-T}$ is the transpose of inverse $\boldsymbol{K}_{T S}$.

RNGA: the normalized gain for control pair $y_{i}-u_{j}$, denoted by $k_{N T S, i j}$, reflects the total effect of $u_{j}$ on $y_{i}$ by including both $k_{T S, i j}$ and $e_{T S, i j}$ as $[18,28]$ :

$$
k_{N T S, i j}=\frac{k_{T S, i j}}{e_{T S, i j}}
$$

Extend Eq. (26) to the overall process to obtain the normalized gain matrix $\boldsymbol{K}_{N T S}$ as [18,28]:

$$
\boldsymbol{K}_{N T S}=\boldsymbol{K}_{T S} \odot \boldsymbol{E}_{T S}
$$

where $\odot$ is element-by-element division. Denote the normalized gain of loop $y_{i}-u_{j}$ when all other control-loops are closed as $\hat{k}_{N T S}, i j$, where $\hat{k}_{N T S, i j}=\hat{k}_{T S, i j} / \hat{e}_{T S, i j}, \hat{e}_{T S, i j}$ is the normalized integrated error of $y_{i}-u_{j}$ when other loops are closed. The relative normalized gain, denoted by $\phi_{T S, i j}$, can be defined as [18,28]:

$$
\phi_{T S, i j}=\frac{k_{N T S, i j}}{\hat{k}_{N T S, i j}}
$$

RNGA is an array derived by collecting all the normalized gains as RNGA $=\left[\phi_{T S, i j}\right]_{n \times n}$, which can be calculated only using individual open-loop information [18,28]:

$$
\mathrm{RNGA}=\boldsymbol{K}_{N T S} \otimes \boldsymbol{K}_{N T S}^{-T}
$$

From RGA and RNGA, the control pairs can be selected according to the following rules [18,28]:

i) All paired RGA and RNGA elements should be positive;

ii) The paired RNGA elements are closest to 1 ;

iii) Large RNGA elements should be avoided;

Place the paired elements on the diagonal positions of $\boldsymbol{K}_{T S}$ through column swap, the value of Niederlinski index (NI) [31], can be calculated as:

$$
\mathrm{NI}=\frac{\operatorname{det}\left[\boldsymbol{K}_{T S}\right]}{\prod_{i=1}^{n} k_{T S, i i}}
$$

where det $\left[\boldsymbol{K}_{T S}\right]$ denotes determinant of $\boldsymbol{K}_{T S}$ after column swap, $\Pi_{i=1}^{n} k_{T S, i i}$ is the product of paired elements. A positive NI is a necessary condition for paired system to be stable [31]. Therefore, an additional rule for pairing is

iv) $\mathrm{NI}>0$

\section{Effective T-S fuzzy model}

The ETSM for a control pair $y_{i}-u_{j}$, denoted by $\hat{f}_{T S, i j}$, is the open-loop T-S fuzzy model for $y_{i}-u_{j}$ when all other control-loops are closed. Thus its steady-state gain and normalized integrated error are $\hat{k}_{T S, i j}$ and $\hat{e}_{T S, i j}$. Since the open-loop model for a certain control pair when other loops are closed will have similar frequency properties to that when all loops are open if the process is well paired [15], it is reasonable 
to keep part of the coefficients of $\hat{f}_{T S, i j}$ same to that of $f_{T S, i j}$. Inspired by the ETF construction proposed in [15], we choose the Type-1 ETSM consisting of following fuzzy rules:

$$
\begin{array}{rc}
\text { Rule } l: \text { IF } & \boldsymbol{x}_{i j}(k) \text { is } \quad C_{i j}^{l} \\
\text { THEN } & y_{i}^{l}(k)=\hat{a}_{i j, 0}^{l} \cdot u_{j}\left(k-\hat{\tau}_{i j}\right)+\hat{a}_{i j, 1}^{l} \cdot u_{j}\left(k-\hat{\tau}_{i j}-1\right)+\cdots+\hat{a}_{i j, p}^{l} \cdot u_{j}\left(k-\hat{\tau}_{i j}-p\right) \\
& +b_{i j, 1}^{l} \cdot y_{i}(k-1)+\cdots+b_{i j, q}^{l} \cdot y_{i}(k-q)
\end{array}
$$

where $\hat{a}_{i j, r}^{l}(r=0,1, \ldots, p)$ and $\hat{\tau}_{i j}$ are the coefficients revised from $a_{i j, r}^{l}(r=0,1, \ldots, p)$ and $\tau_{i j}$ of the individual open-loop Type-1 T-S fuzzy model as in Eq. (4). Similarly, we choose the Type-2 ETSM consisting of following fuzzy rules:

$$
\begin{array}{cc}
\text { Rule } l: \text { IF } & \boldsymbol{x}_{i j}(k) \text { is } \quad \tilde{C}_{i j}^{l} \\
\text { THEN } & \tilde{y}_{i}^{l}(k)=\hat{\tilde{a}}_{i j, 0}^{l} \cdot u_{j}\left(k-\hat{\tau}_{i j}\right)+\hat{\tilde{a}}_{i j, 1}^{l} \cdot u_{j}\left(k-\hat{\tau}_{i j}-1\right)+\cdots+\hat{\tilde{a}}_{i j, p}^{l} \cdot u_{j}\left(k-\hat{\tau}_{i j}-p\right) \\
& +\tilde{b}_{i j, 1}^{l} \cdot y_{i}(k-1)+\cdots+\tilde{b}_{i j, q}^{l} \cdot y_{i}(k-q)
\end{array}
$$

where $\hat{\tilde{a}}_{i j, r}^{l}=\left[\hat{a}_{i j, l b, r}^{l}, \quad \hat{a}_{i j, r b, r}^{l}\right]$ and $\hat{\tau}_{i j}$ are revised from $\tilde{a}_{i j, r}^{l}=\left[a_{i j, l b, r}^{l}, \quad a_{i j, r b, r}^{l}\right]$ and $\tau_{i j}$ of the individual open-loop Type-2 T-S fuzzy model as in Eq. (6).

The quantified interacting effects on steady-state gain of $y_{i}-u_{j}$ can be derived from relative gain $\lambda_{T S, i j}=k_{T S, i j} / \hat{k}_{T S, i j}$, while the quantified interacting effects on dynamic property can be derived from both relative gain $\lambda_{T S, i j}$ and relative normalized gain $\phi_{T S, i j}$ by [17]:

$$
\frac{\phi_{T S, i j}}{\lambda_{T S, i j}}=\frac{\hat{e}_{T S, i j}}{e_{T S, i j}} \equiv \gamma_{T S, i j}
$$

where $\gamma_{T S, i j}$ is the relative normalized integrated error [17]. For the whole process we have:

$$
\boldsymbol{\Gamma}_{\mathrm{TS}}=\left[\gamma_{\mathrm{TS}, i j}\right]_{n \times n}=\mathrm{RNGA} \odot \mathrm{RGA}
$$

Based on $\lambda_{T S, i j}$ and $\gamma_{T S, i j}$, the revised coefficients of Type- 1 and the Type-2 ETSM can be calculated as follows:

According to Eq. (21), the steady-state gain $\hat{k}_{T S, i j}$ of an ETSM $\hat{f}_{T S, i j}$ based on a given operating point $\boldsymbol{x}_{0, i j}$ can be calculated as:

$$
\hat{k}_{T S, i j}=\frac{\hat{a}_{i j, 0}+\hat{a}_{i j, 1}+\cdots+\hat{a}_{i j, p}}{1-\left(b_{i j, 1}+b_{i j, 2}+\cdots+b_{i j, q}\right)}
$$

For a Type-1 ETSM, the coefficients $\hat{a}_{i j, r}(r=0,1, \ldots, p)$ in Eq. (35) are calculated by

$$
\hat{a}_{i j, r}=\sum_{l=1}^{M_{i j}} \mu_{i j}^{l}\left(\boldsymbol{x}_{0, i j}\right) \hat{a}_{i j, r}^{l}
$$

Submitting Eqs. (21) and (24) into Eq. (35) to have the following equation to determine $\hat{a}_{i j, r}^{l}$ :

$$
\hat{a}_{i j, r}^{l}=\frac{a_{i j, r}^{l}}{\lambda_{T S, i j}}
$$

For a Type-2 ETSM, the coefficients $\hat{a}_{i j, r}(r=0,1, \ldots, p)$ in Eq. (35) are determined by

$$
\hat{a}_{i j, r}=\frac{\hat{a}_{i j, l b, r}+\hat{a}_{i j, r b, r}}{2}
$$

where $\hat{a}_{i j, l b, r}=\sum_{l=1}^{M_{i j}} \mu_{i j, l b}^{l}\left(\boldsymbol{x}_{0, i j}\right) \cdot \hat{a}_{i j, l b, r}^{l} / \sum_{l=1}^{M_{i j}} \mu_{i j, l b}^{l}\left(\boldsymbol{x}_{0, i j}\right)$ and $\hat{a}_{i j, r b, r}=\sum_{l=1}^{M_{i j}} \mu_{i j, r b}^{l}\left(\boldsymbol{x}_{0, i j}\right) \cdot \hat{a}_{i j, r b, r}^{l} / \sum_{l=1}^{M_{i j}} \mu_{i j, r b}^{l}\left(\boldsymbol{x}_{0, i j}\right)$. Submitting Eqs. (20), (21),

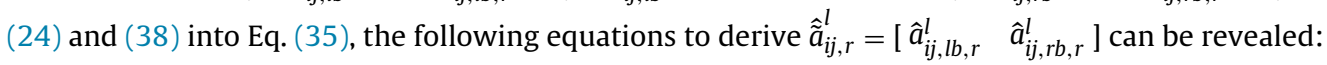

$$
\hat{a}_{i j, l b, r}^{l}=\frac{a_{i j, l b, r}^{l}}{\lambda_{T S, i j}}, \quad \hat{a}_{i j, r b, r}^{l}=\frac{a_{i j, r b, r}^{l}}{\lambda_{T S, i j}}
$$

According to Eq. (22), the normalized integrated error $\hat{e}_{T S, i j}$ of a Type- 1 or Type-2 ETSM $\hat{f}_{T S, i j}$ based on the given operating point $\boldsymbol{x}_{0, i j}$ is computed by:

$$
\hat{e}_{T S, i j}=\frac{\sum_{r=0}^{p} r \hat{a}_{i j, r}-\sum_{w=0}^{p} \sum_{s=1}^{q} \hat{a}_{i j, w} \cdot b_{i j, s} \cdot|w-s| \cdot \operatorname{sgn}(w-s)}{\left(\hat{a}_{i j, 0}+\hat{a}_{i j, 1}+\cdots+\hat{a}_{i j, p}\right)\left(1-\left(b_{i j, 1}+\cdots+b_{i j, q}\right)\right)} \cdot T+\hat{\tau}_{i j} \cdot T
$$

For a Type-1 ETSM, $\hat{a}_{i j, r}$ is determined by Eq. (36) and $b_{i j, s}$ is determined by Eq. (19). While for a Type-2 ETSM, $\hat{a}_{i j, r}$ is determined by Eq. (38) and $b_{i j, s}$ is determined by Eq. (20). Submitting Eqs. (22), (33), (37) / (39) into (40), after arrangement, gives the following equation to calculate $\hat{\tau}_{i j}$ :

$$
\hat{\tau}_{i j}=\frac{\sum_{r=0}^{p} r a_{i j, r}-\sum_{w=0}^{p} \sum_{s=1}^{q} a_{i j, w} \cdot b_{i j, s} \cdot|w-s| \cdot \operatorname{sgn}(w-s)}{\left(a_{i j, 0}+a_{i j, 1}+\cdots+a_{i j, p}\right)\left(1-\left(b_{i j, 1}+\cdots+b_{i j, q}\right)\right)} \cdot\left(\gamma_{T S, i j}-1\right)+\tau_{i j} \cdot \gamma_{T S, i j}
$$




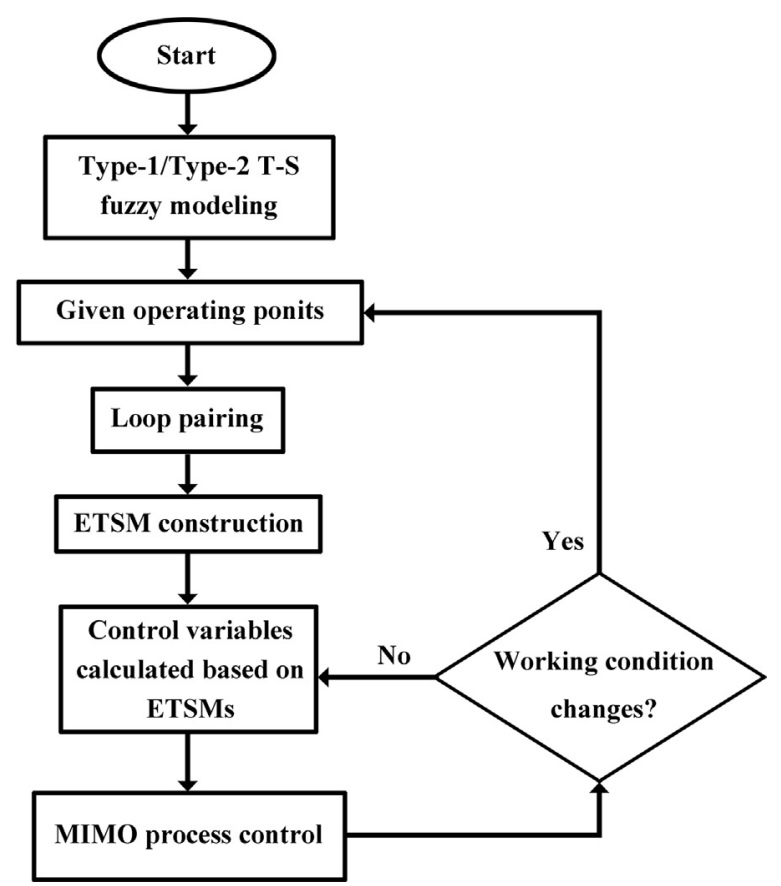

Fig. 3. The working procedure for ETSM based decentralized controller design.

Several experimental results demonstrate that for well paired MIMO processes, the values of $\gamma_{T S, i j}$ 's of paired control-loops are closed to 1 . Thus Eq. (41) can be simplified as:

$$
\hat{\tau}_{i j} \approx \tau_{i j} \cdot \gamma_{T S, i j}
$$

Eqs. (37), (39) and (42) provide simple calculations to revise the coefficients to describe interacting effects. However, an important and necessary fact which can not be ignored is that a control system should possess integrity property [15,17], which means, the system should remain stable whether other loops are put in or taken out. Moreover, the integrity requires that when controlling a certain loop after all other loops remove, the performance of the controller designed based on the ETSM should be no more aggressive than that of the controller designed based on the individual open-loop model [17]. Note that larger absolute value of steady-state gain and larger time delay imply more challenges for a stable control system design. In a bid to maintain the integrity property, an ETSM should choose the coefficients between original and revised ones that can reflect "worse condition" for controller design. Therefore, we have the following criterion to determine $\hat{a}_{i j, r}, \hat{\tilde{a}}_{i j, r}$ and $\hat{\tau}_{i j}$ for Type-1/Type-2 ETSMs:

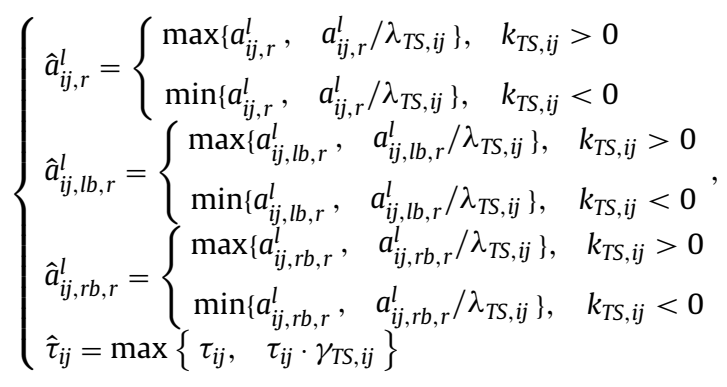

Based on ETSMs, linear SISO control algorithms can be directly applied to design decentralized controllers for nonlinear MIMO processes. The steps to devise the ETSM based decentralized controllers are summarized as follows with a flowchart given in Fig. 3.

i) For an $n \times n$ process, collect data samples from each input-output channel to build an individual Type-1 or Type-2 open-loop T-S fuzzy model to form an $n \times n$ Type-1 or Type-2 fuzzy model matrix $\boldsymbol{F}_{T S}$.

ii) At a certain working condition, calculated steady-state gain $k_{T S, i j}$ and normalized integrated error $e_{T S, i j}$ for each individual element in $\boldsymbol{F}_{T S}$ to obtain $\boldsymbol{K}_{T S}$ and $\boldsymbol{E}_{T S}$.

iii) Use RNGA based criterion to pair inputs and outputs to determine a decentralized control configuration.

iv) For each control pair, revise the coefficients of its individual Type-1 or Type-2 open-loop T-S fuzzy model according to Eq. (43) to obtain a Type-1 or a Type-2 ETSM. Afterwards, design a local controller based on each ETSM to achieve a decentralized control system.

v) If the working condition changes, repeat step ii)-iv). 


\section{Case studies}

\subsection{Simulations}

Consider a three-input-three-output nonlinear process [19]:

$$
\left\{\begin{array}{l}
\dot{x}_{1}=x_{2}+5 x_{1}^{2} x_{2}+6 x_{2}^{2} \\
\dot{x}_{2}=-4 x_{1}-5 x_{2}+8 x_{1} x_{2}+u_{1} \\
\dot{x}_{3}=x_{4} \\
\dot{x}_{4}=-6 x_{3}-5 x_{4}+3 x_{3}^{3}+10 x_{3} x_{4} x_{5}+u_{2} \\
\dot{x}_{5}=x_{6}+4 x_{7}^{2} \\
\dot{x}_{6}=x_{7}+5 x_{5} x_{6}^{2} x_{7} \\
\dot{x}_{7}=-14 x_{5}-23 x_{6}-10 x_{7}+7 x_{5} x_{6} x_{7}+u_{3} \\
y_{1}=5 x_{1}+5 x_{2}+6 x_{3}+2 x_{4}+14 x_{5}+9 x_{6}+x_{7} \\
y_{2}=8 x_{1}+2 x_{2}+3 x_{3}+4 x_{5}+6 x_{6}+2 x_{7} \\
y_{3}=x_{1}+x_{2}+4 x_{3}+2 x_{4}+1.4 x_{5}+0.2 x_{6}
\end{array}\right.
$$

where $x_{r}$ 's $(r=1, \ldots, 7)$ are state variables. The time delays in this process are $\tau_{i 1}^{\prime}=\tau_{i 2}^{\prime}=2$ (sec) and $\tau_{i 3}^{\prime}=1$ (sec) for $i=1,2,3$. Choose the sampling interval as $T=0.1 \mathrm{sec}$, suppose there are disturbances random but bounded in $[-0.2,0.2]$ on the inputs of the sampled data pairs, construct a Type- 1 and a Type- 2 fuzzy model with $p=0$ and $q=2$ for each input-output channel (the results are shown in Appendix A). Given the operating points as $\boldsymbol{x}_{0, i j}=\left[\begin{array}{lll}u_{0, j}\left(k_{0}-\tau_{i j}\right) & y_{0, i}\left(k_{0}-1\right) & y_{0, i}\left(k_{0}-2\right)\end{array}\right]=\left[\begin{array}{lll}0 & 0 & 0\end{array}\right]$ for $i, j=1,2,3$, from the Type-1 T-S fuzzy models, the following results can be obtained:

$$
\begin{aligned}
& \boldsymbol{K}_{T S}=\left[\begin{array}{lll}
1.2565 & 0.9784 & 1.0782 \\
2.1238 & 0.5486 & 0.2905 \\
0.2493 & 0.6743 & 0.1313
\end{array}\right], \quad \boldsymbol{E}_{T S}=\left[\begin{array}{lll}
2.1954 & 2.5234 & 2.1538 \\
2.8221 & 3.5756 & 1.1021 \\
2.1872 & 2.3181 & 6.9247
\end{array}\right] \\
& R G A=\left[\begin{array}{ccc}
-0.1498 & -0.1944 & 1.3442 \\
1.2235 & -0.0548 & -0.1687 \\
-0.0737 & 1.2492 & -0.1755
\end{array}\right], \quad \text { RNGA }=\left[\begin{array}{ccc}
-0.6522 & 0.0945 & 1.5577 \\
1.6075 & -0.1095 & -0.4980 \\
-0.0447 & 1.0150 & -0.0598
\end{array}\right]
\end{aligned}
$$

According to RNGA based criterion, the decentralized control configuration can be determined $y_{1}-u_{3} / y_{2}-u_{1} / y_{3}-u_{2}, \mathrm{NI}=0.9598>0$, and the normalized integrated error matrix is:

$$
\boldsymbol{\Gamma}_{T S}=\left[\begin{array}{ccc}
4.3545 & -0.4861 & 1.1589 \\
1.3138 & 1.9981 & 2.9520 \\
-0.6065 & 0.8125 & 0.3405
\end{array}\right]
$$

The results derived from the Type-2 T-S fuzzy models are:

$$
\begin{aligned}
& \boldsymbol{K}_{T S}=\left[\begin{array}{lll}
1.2502 & 0.9762 & 1.0750 \\
2.1205 & 0.5480 & 0.2902 \\
0.2481 & 0.6724 & 0.1316
\end{array}\right], \quad \boldsymbol{E}_{T S}=\left[\begin{array}{lll}
2.1939 & 2.5221 & 2.1479 \\
2.8188 & 3.5633 & 1.1018 \\
2.1847 & 2.3172 & 6.8859
\end{array}\right] \\
& R G A=\left[\begin{array}{ccc}
-0.1492 & -0.1961 & 1.3453 \\
1.2228 & -0.0543 & -0.1685 \\
-0.0736 & 1.2504 & -0.1768
\end{array}\right], \quad \text { RNGA }=\left[\begin{array}{ccc}
-0.6478 & 0.0930 & 1.5548 \\
1.6039 & -0.1093 & -0.4946 \\
0.0439 & 1.0163 & -0.0602
\end{array}\right]
\end{aligned}
$$

The decentralized control pairs selected by the RNGA based criterion is same to that derived from Type- 1 fuzzy models: $y_{1}-u_{3} / y_{2}-u_{1} / y_{3}-u_{2}$, where $\mathrm{NI}=0.9596>0$, and

$$
\boldsymbol{\Gamma}_{\text {TS }}=\left[\begin{array}{ccc}
4.3415 & -0.4742 & 1.1557 \\
1.3117 & 2.0119 & 2.9356 \\
-0.5960 & 0.8127 & 0.3403
\end{array}\right]
$$

The gain and phase margins based control algorithm employed in [15,17] is selected to design controllers based on Type-1 and Type-2 ETSMs (The details are given in Appendix A), and the required gain and phase margins for the ETSM based control system are set as 3 and $\pi / 3$. For comparison, we linearize the functions of Eq. (44) at the given operating points to obtain a transfer function matrix to apply the RNGA based ETF method [17] using the same control algorithm with the same required gain and phase margins (the details are also given in Appendix A). Let the reference values be $r v_{1}=1.5, r v_{2}=1$ and $r v_{3}=0$, the control performances are shown in Fig. 4 .

As can be seen in Fig. 4, when given the same gain and phase margin requirements, the controllers based on fuzzy models built from data with inexactness can achieve smaller overshoots compared to that based on transfer functions linearized from exact mathematical model. 

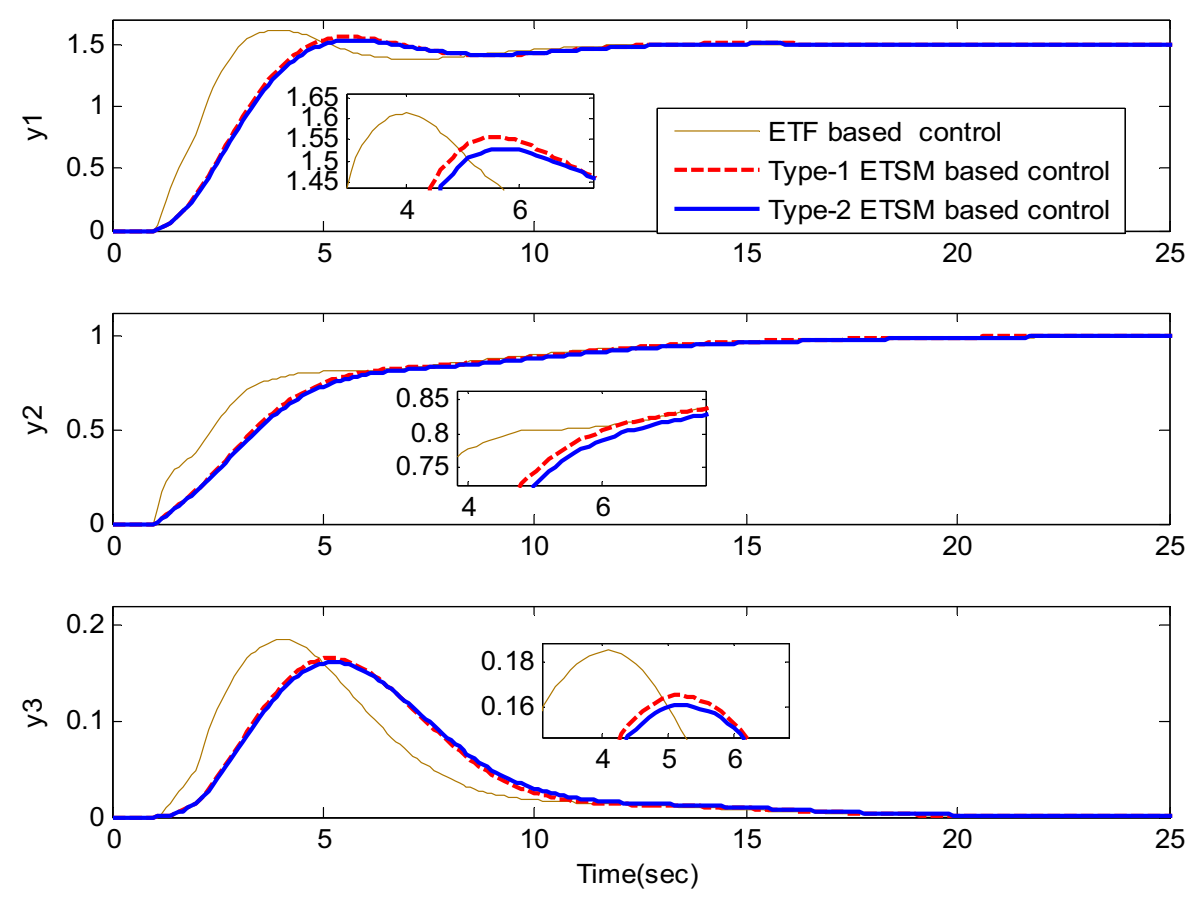

Fig. 4. The comparisons of ETF and ETSM based control for Eq. (44).

Table 1

Performance indexes of Type-1 and Type-2 ETSM based control for Eq. (44).

\begin{tabular}{|c|c|c|c|c|c|c|c|c|c|c|c|c|}
\hline & \multicolumn{3}{|l|}{ IAE } & \multicolumn{3}{|l|}{ ISE } & \multicolumn{3}{|l|}{ ITAE } & \multicolumn{3}{|l|}{ ITSE } \\
\hline & $y_{1}$ & $y_{2}$ & $y_{3}$ & $y_{1}$ & $y_{2}$ & $y_{3}$ & $y_{1}$ & $y_{2}$ & $y_{3}$ & $y_{1}$ & $y_{2}$ & $y_{3}$ \\
\hline Type-1 & 4.83 & 4.99 & 0.90 & 5.49 & 2.99 & 0.10 & 10.81 & 21.81 & 6.08 & 7.19 & 5.97 & 0.54 \\
\hline Type-2 & 4.77 & 4.81 & 0.89 & 5.42 & 2.91 & 0.10 & 10.56 & 19.89 & 5.83 & 6.97 & 5.59 & 0.54 \\
\hline
\end{tabular}

Table 2

Performance indexes of Type-1 and Type-2 ETSM based control for Case-I and Case-II.

\begin{tabular}{|c|c|c|c|c|c|c|c|c|c|c|c|c|c|}
\hline & & \multicolumn{3}{|l|}{ IAE } & \multicolumn{3}{|l|}{ ISE } & \multicolumn{3}{|l|}{ ITAE } & \multicolumn{3}{|l|}{ ITSE } \\
\hline & & $y_{1}$ & $y_{2}$ & $y_{3}$ & $y_{1}$ & $y_{2}$ & $y_{3}$ & $y_{1}$ & $y_{2}$ & $y_{3}$ & $y_{1}$ & $y_{2}$ & $y_{3}$ \\
\hline \multirow[t]{2}{*}{ Case-I } & Type-1 & 8.71 & 4.84 & 2.59 & 7.63 & 2.71 & 0.78 & 57.18 & 27.05 & 20.90 & 21.30 & 5.74 & 4.21 \\
\hline & Type-2 & 8.15 & 4.60 & 2.42 & 6.73 & 2.67 & 0.73 & 48.90 & 23.33 & 18.55 & 18.44 & 5.21 & 3.92 \\
\hline \multirow[t]{2}{*}{ Case-II } & Type-1 & 33.2 & 17.8 & 11.2 & 21.4 & 6.6 & 2.8 & 1077.5 & 562.3 & 372.4 & 328.4 & 89.4 & 41.7 \\
\hline & Type-2 & 24.7 & 13.5 & 8.33 & 16.2 & 5.2 & 2.1 & 586.5 & 307.3 & 204.7 & 182.3 & 50.2 & 24.5 \\
\hline
\end{tabular}

The performances of Type- 1 and Type- 2 ETSM based control are camparable in this case. In a bid to explicitly demonstrate their differences, the comparisions of four performance indexes, IAE $=\sum_{k=0}^{\infty}\left|r v_{i}-y_{i}(k)\right| \cdot T, \operatorname{ISE}=\sum_{k=0}^{\infty}\left(r v_{i}-y_{i}(k)\right)^{2} \cdot T, \operatorname{ITAE}=\sum_{k=0}^{\infty} k \cdot\left|r v_{i}-y_{i}(k)\right| \cdot T$ and ITSE $=\sum_{k=0}^{\infty} k \cdot\left(r v_{i}-y_{i}(k)\right)^{2} \cdot T$, between Type-1 and Type-2 ETSM based control are employed and shown in Table 1, which prove Type-2 control can achieve smaller integrated errors.

Suppose the third state equation $\dot{x}_{3}=x_{4}$ in Eq. (44) is changed to the following two cases due to the uncertainties:

Case-I: $\dot{x}_{3}=x_{4}+1.5 u_{1}$; Case-II: $\dot{x}_{3}=x_{4}+2.31 u_{1}$

Apply the controllers to the changed processes, the comparisons are shown in Fig. 5, and the four indexes of ETSM based control are given in Table 2 .

As can be seen in Fig. 5 and Table 2, ETSM based control can provide much better results than ETF based control, and Type-2 ETSM based control can achieve smaller overshoots, less settling time and smaller integrated errors compared to its Type-1 counterpart. When the changed coefficient becomes larger due to the increased degree of uncertainty as:

Case-III: $\dot{x}_{3}=x_{4}+2.5 u_{1}$

The process under the ETF based control becomes instable while its outputs under the ETSM based control can still reach the reference values as shown in Fig. 6. The four indexes of ETSM based control in Table 3 prove that Type-2 ETSM based control is more robust.

Concluded from Fig. 4-6 and Table 1-3, ETSM based control can give better performances when compared to their ETF based counterparts in every case. As the degree of uncertainty become lager, Type-2 ETSM based control can provide more satisfactory results than Type-1 ETSM based control in terms of robustness. 

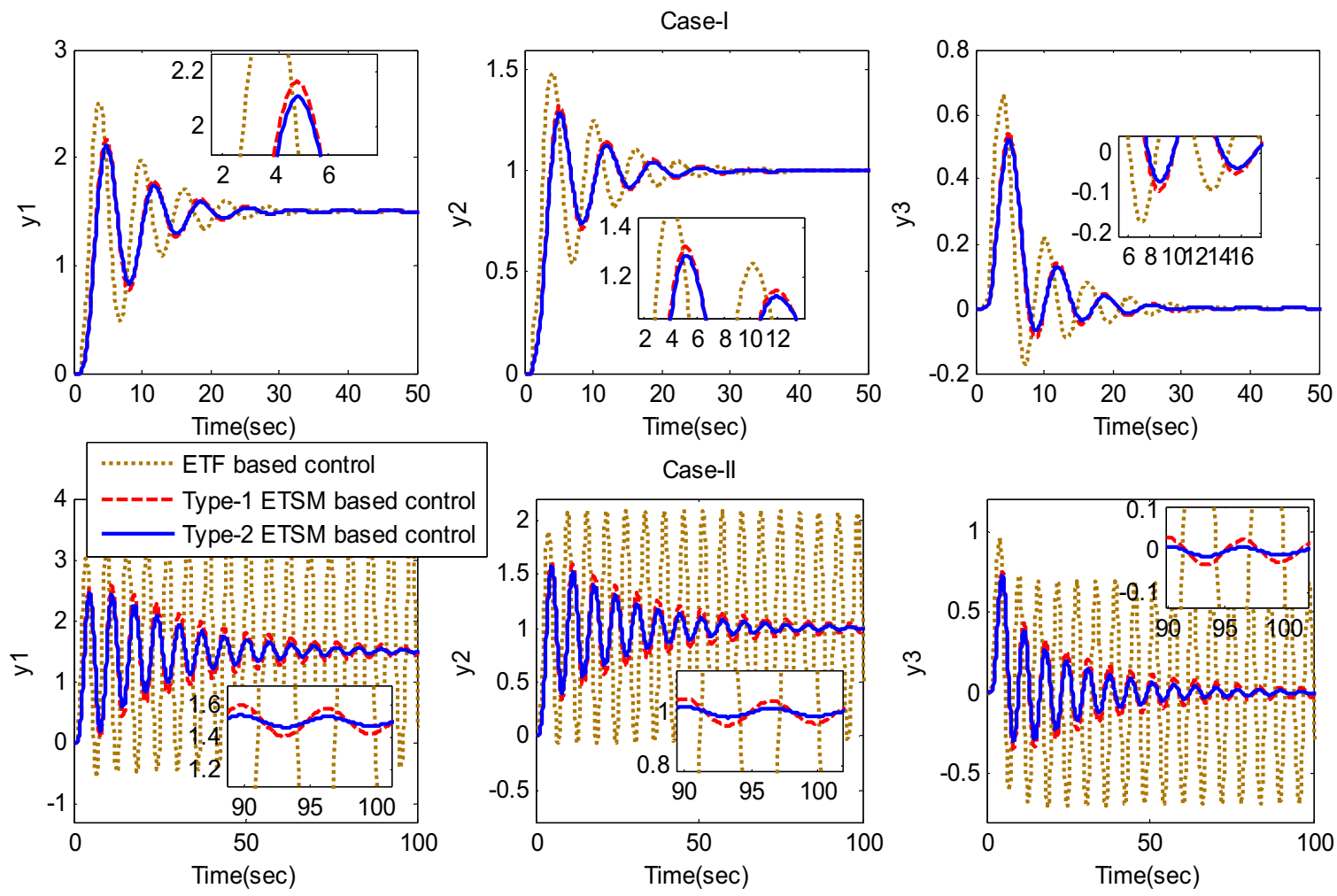

Fig. 5. Comparisons of ETF and ETSM based control for Case-I and Case-II.
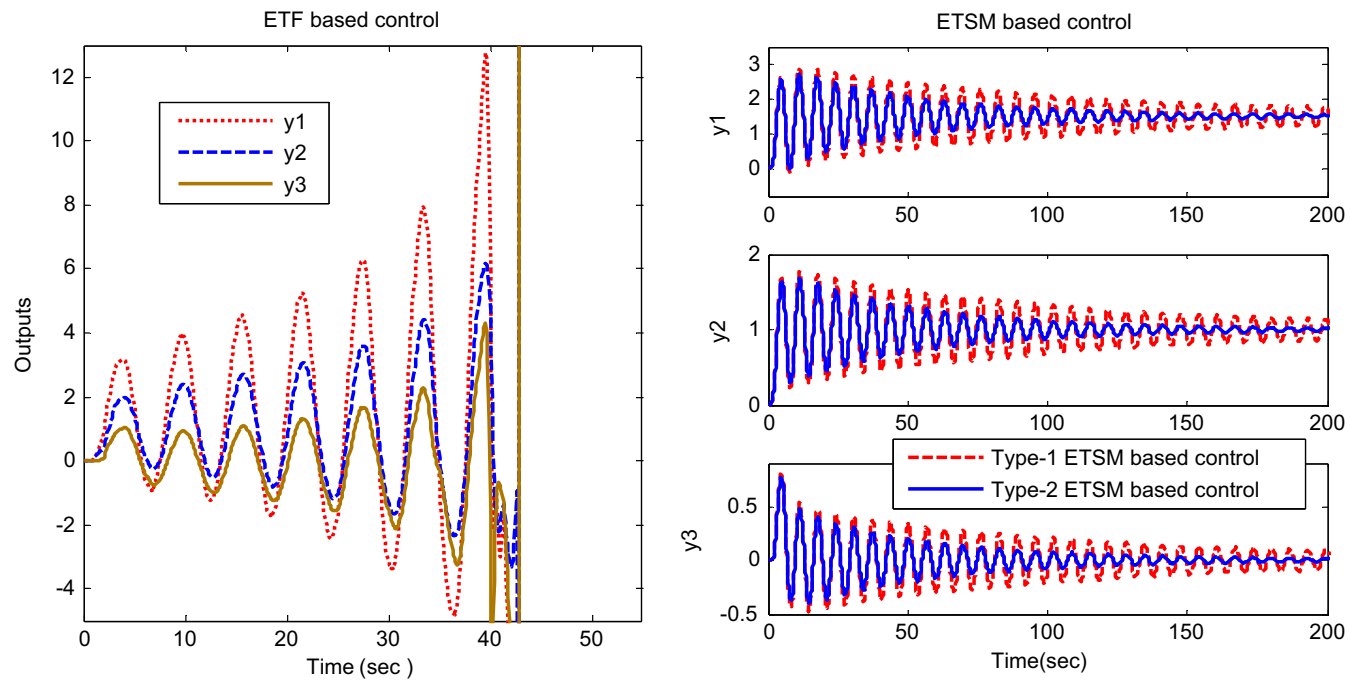

Fig. 6. Comparisons of ETF and ETSM based control for Case-III.

Table 3

Performance indexes of Type- 1 and Type-2 ETSM based control for Case-III.

\begin{tabular}{|c|c|c|c|c|c|c|c|c|c|c|c|c|}
\hline & \multicolumn{3}{|l|}{ IAE } & \multicolumn{3}{|l|}{ ISE } & \multicolumn{3}{|l|}{ ITAE } & \multicolumn{3}{|l|}{ ITSE } \\
\hline & $y_{1}$ & $y_{2}$ & $y_{3}$ & $y_{1}$ & $y_{2}$ & $y_{3}$ & $y_{1}$ & $y_{2}$ & $y_{3}$ & $y_{1}$ & $y_{2}$ & $y_{3}$ \\
\hline Type-1 & 116 & 62.4 & 40.4 & 67.5 & 19.8 & 8.51 & 1457.1 & 7758 & 5079 & 4051 & 1145 & 495.4 \\
\hline Type-2 & 53.1 & 28.9 & 18.4 & 32.4 & 9.96 & 4.24 & 296.1 & 1582 & 1038 & 832.4 & 237.0 & 105.0 \\
\hline
\end{tabular}

\subsection{Application in a multi-evaporator refrigeration system}

An experimental multi-evaporator refrigeration system with three evaporators (EVAP1, EVAP2 and EVAP3) is shown in Fig. 7, and its schematic diagram and pressure $(\mathrm{P})$-enthalpy $(\mathrm{h})$ chart are shown in Fig. 8. In this system, R134a is used as the refrigerant. For EVAP1, water is used as heat transfer fluid to convey the cooling to meet the air-conditioning requirements. While for EVAP 2 used for perishable food 


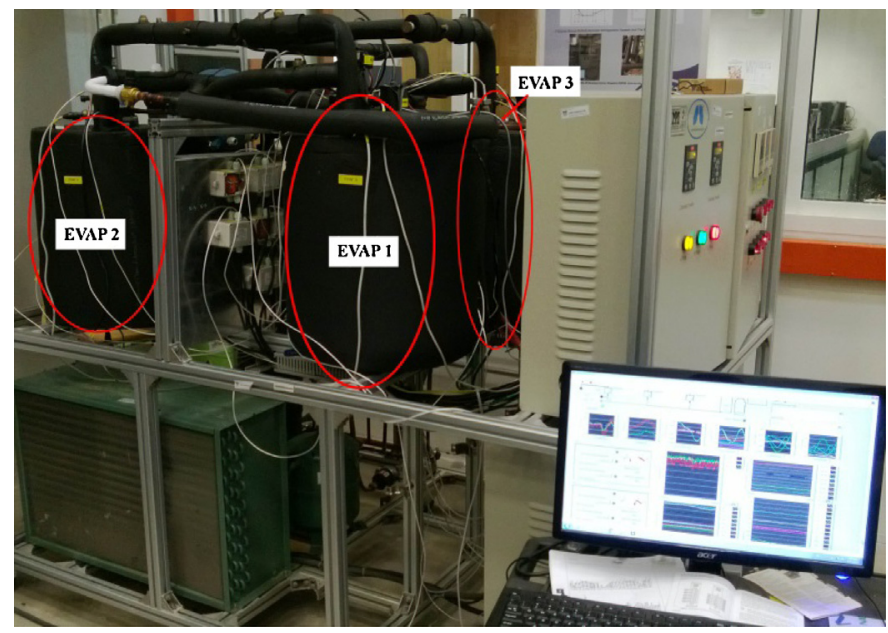

Fig. 7. The experimental multi-evaporator refrigeration system.

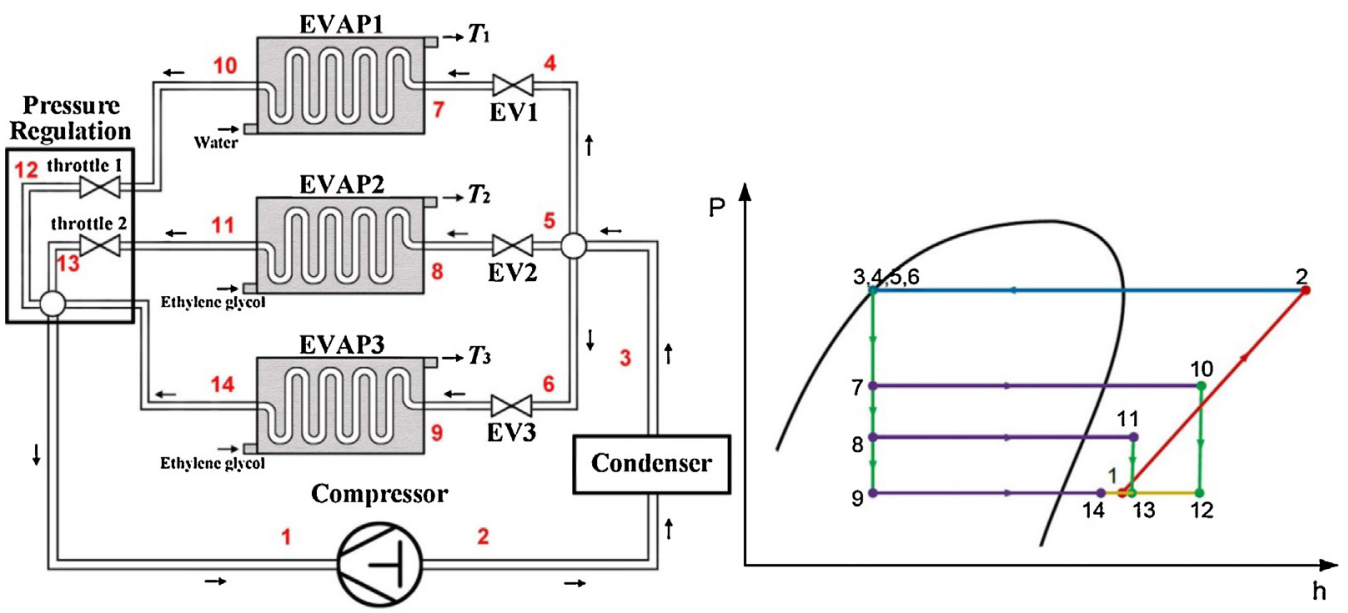

Fig. 8. The schematic diagram and pressure (P)-enthalpy (h) chart of the multi-evaporator refrigeration system.

storage and EVAP3 used for freezing where evaporating temperatures are low that water may be frozen, ethylene glycol solution is used instead. The working process as shown in Fig. 8 is: the refrigerant as a saturated vapor with a low pressure (state $\mathbf{1}$ ) enters the compressor and is compressed isentropically to a superheated vapor with a high pressure (state 2 ). Then it enters into the condenser where it is cooled and condensed into liquid phase (state $\mathbf{3}$ ) by rejecting heat to the external environment. Afterwards it is divided into three flows (states 4, 5 and 6), which go into EVAP1 (state 7), EVAP2 (state 8) and EVAP3 (state 9) after their pressures are reduced through three expansion valves (EV1, EV2, and EV3) respectively. By absorbing heats from the ambient environments of the evaporators, the three flows evaporate at specified temperatures to become saturated vapor (states 10,11 and 14). In the pressure regulation device, the flows at state $\mathbf{1 0}$ and $\mathbf{1 1}$ are throttled to state $\mathbf{1 2}$ and $\mathbf{1 3}$ respectively such that their pressures are equal to that of the flow at state $\mathbf{1 4}$ from EVAP3 which has the lowest evaporating pressure and temperature. Finally, the three flows mix up into one at state $\mathbf{1}$ and return to compressor to complete the refrigeration cycle.

In the experiment, the compressor power and the speeds of fans are fixed. The flow rates of refrigerant in three evaporators can be adjusted to satisfy different cooling loads through regulating the opening degrees of three EVs. The opening change in any one of the EVs will have impacts on three refrigerant flow rates of three evaporators subsequently affect the temperatures of heat transfer fluids $T_{1}$, $T_{2}$ and $T_{3}$ as shown in Fig. 8. Therefore, an interconnected nonlinear three-input-three-output $(3 \times 3)$ process can be formed where the three EV opening degrees are used to regulate the heat transfer fluid temperatures of three evaporators. Since the designed working condition for this multi-evaporator refrigeration system is $T_{1, d}=17^{\circ} \mathrm{C}, T_{2, d}=3^{\circ} \mathrm{C}$ and $T_{3, d}=-8^{\circ} \mathrm{C}$ with the corresponding EV opening degrees as $85 \%, 43 \%$ and $16 \%$ respectively, let the outputs of this $3 \times 3$ process be $y_{i}=T_{i}-T_{i, d}(i=1,2,3)$, and the opening ranges of EV1, EV2 and EV3 be $[70 \%, 100 \%],[31 \%, 55 \%]$ and $[12 \%, 20 \%]$ which are uniformly scaled to $[-3,3]$ to be the variation ranges of inputs $u_{j}(j=1,2,3)$ for constructing fuzzy models. The step responses for this $3 \times 3$ process are shown in Fig. 9 .

The time delay can be measured as: $\tau_{11}^{\prime}=1(\mathrm{~min}), \tau_{12}^{\prime}=1.6(\mathrm{~min}), \tau_{13}^{\prime}=1.5(\mathrm{~min}), \tau_{21}^{\prime}=1.4(\mathrm{~min}), \tau_{22}^{\prime}=1.2(\mathrm{~min}), \tau_{23}^{\prime}=$ $1.4(\mathrm{~min}), \tau_{31}^{\prime}=1.4(\mathrm{~min}), \tau_{32}^{\prime}=1.5(\mathrm{~min})$ and $\tau_{33}^{\prime}=1.2(\mathrm{~min})$, and the sampling interval is chosen as $T=0.5$ (min). Based on the data samples, both Type- 1 and Type- 2 fuzzy models can be constructed for this $3 \times 3$ process as shown in Appendix B. The experiment is carried 

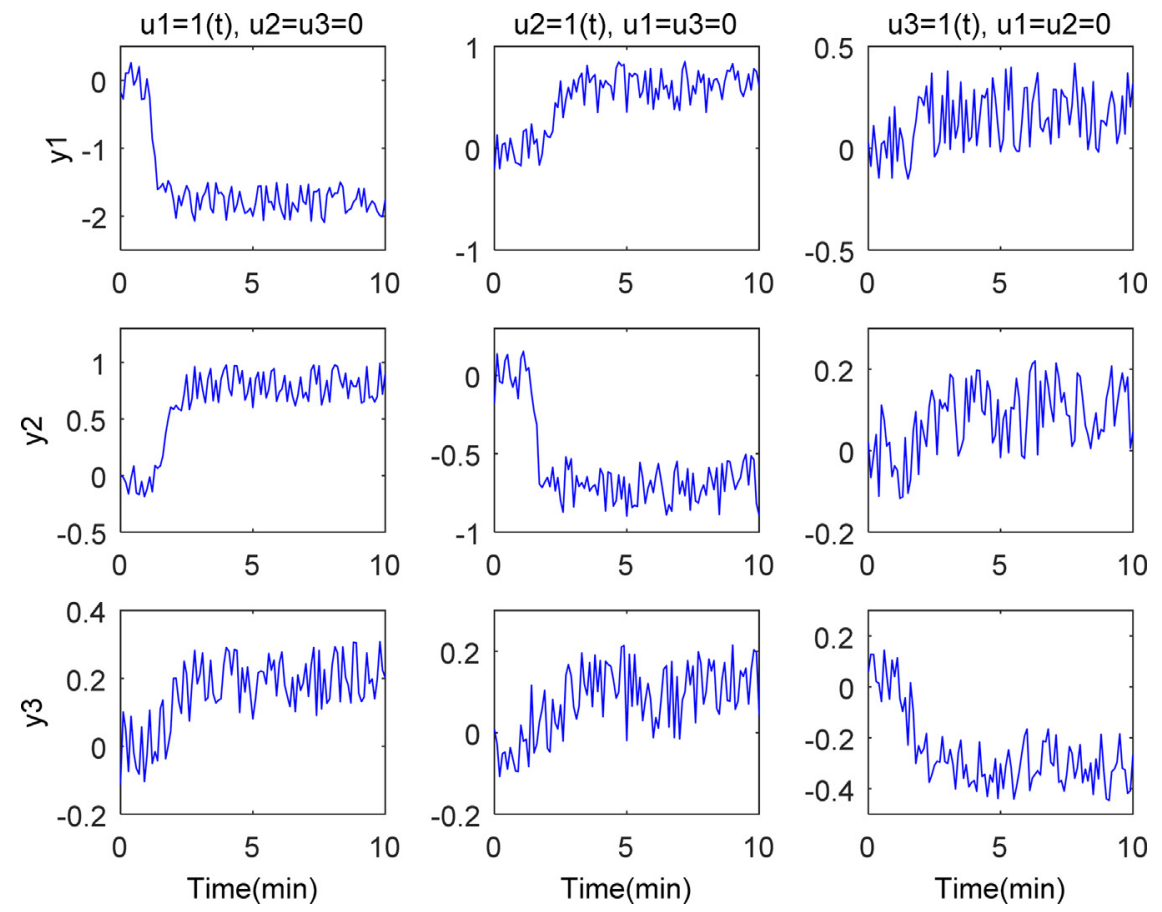

Fig. 9. Step responses for the $3 \times 3$ process.

out in the area of designed working condition around the operating points $\boldsymbol{x}_{0, i j}=\left[\begin{array}{lll}u_{0, j}\left(k_{0}-\tau_{i j}\right) & y_{0, i}\left(k_{0}-1\right) & y_{0, i}\left(k_{0}-2\right)\end{array}\right]=\left[\begin{array}{lll}0 & 0 & 0\end{array}\right]$ for $i, j=1,2$, 3. From Type- 1 fuzzy models, the following results can be obtained:

$$
\begin{aligned}
& \boldsymbol{K}_{T S}=\left[\begin{array}{ccc}
-1.7958 & 0.6011 & 0.2011 \\
0.7983 & -0.6962 & 0.0996 \\
0.2005 & 0.0993 & -0.2961
\end{array}\right], \quad \boldsymbol{E}_{T S}=\left[\begin{array}{cccc}
1.0409 & 1.8491 & 1.7683 \\
1.5136 & 1.3125 & 1.6252 \\
1.5769 & 1.6966 & 1.4837
\end{array}\right] \\
& R G A=\left[\begin{array}{ccc}
2.2836 & -0.9984 & -0.2852 \\
-1.0239 & 2.2168 & -0.1929 \\
-0.2597 & -0.2184 & 1.4780
\end{array}\right], \quad \text { RNGA }=\left[\begin{array}{ccc}
1.3730 & -0.2860 & -0.0870 \\
-0.2936 & 1.3614 & -0.0679 \\
-0.0794 & -0.0755 & 1.1549
\end{array}\right] \\
& \boldsymbol{\Gamma}_{T S}=\left[\begin{array}{lll}
0.6012 & 0.2864 & 0.3051 \\
0.2867 & 0.6141 & 0.3519 \\
0.3058 & 0.3456 & 0.7813
\end{array}\right]
\end{aligned}
$$

The decentralized control structure is $y_{1}-u_{1} / y_{2}-u_{2} / y_{3}-u_{3}$, where $\mathrm{NI}=0.4169>0$. From Type-2 fuzzy models, the results are:

$$
\begin{aligned}
& \boldsymbol{K}_{T S}=\left[\begin{array}{ccc}
-1.7995 & 0.6000 & 0.2009 \\
0.7968 & -0.7012 & 0.0994 \\
0.1997 & 0.0990 & -0.2970
\end{array}\right], \quad \boldsymbol{E}_{T S}=\left[\begin{array}{llll}
1.0427 & 1.8483 & 1.7692 \\
1.5118 & 1.3163 & 1.6258 \\
1.5762 & 1.6959 & 1.4844
\end{array}\right] \\
& R G A=\left[\begin{array}{ccc}
2.2432 & -0.9669 & -0.2763 \\
-0.9916 & 2.1777 & -0.1861 \\
-0.2516 & -0.2108 & 1.4624
\end{array}\right], \quad \text { RNGA }=\left[\begin{array}{ccc}
1.3688 & -0.2829 & -0.0859 \\
-0.2905 & 1.3573 & -0.0668 \\
-0.0784 & -0.0743 & 1.1527
\end{array}\right] \\
& \boldsymbol{\Gamma}_{T S}=\left[\begin{array}{lll}
0.6102 & 0.2926 & 0.3109 \\
0.2929 & 0.6233 & 0.3589 \\
0.3115 & 0.3526 & 0.7882
\end{array}\right]
\end{aligned}
$$

The decentralized control structure is $y_{1}-u_{1} / y_{2}-u_{2} / y_{3}-u_{3}$, same as that obtained from Type- 1 fuzzy models, NI $=0.4247>0$. Using the gain and phase margins based control method to devise the local controller, given the required gain and phase margins are 4 and $3 \pi / 8$ respectively, the performances of Type- 1 and Type-2 ETSM based decentralized control for this multi-evaporator refrigeration system are shown in Fig. 10.

It can be seen from Fig. 10 that the outputs under both Type- 1 and Type-2 ETSM based control can track their reference values in spite of disturbances. Their performance indexes integrated $k=6$ to $k=60 / T$ are compared in Table 4, which demonstrate that Type- 2 ETSM based control can achieve smaller integrated errors in real applications. 

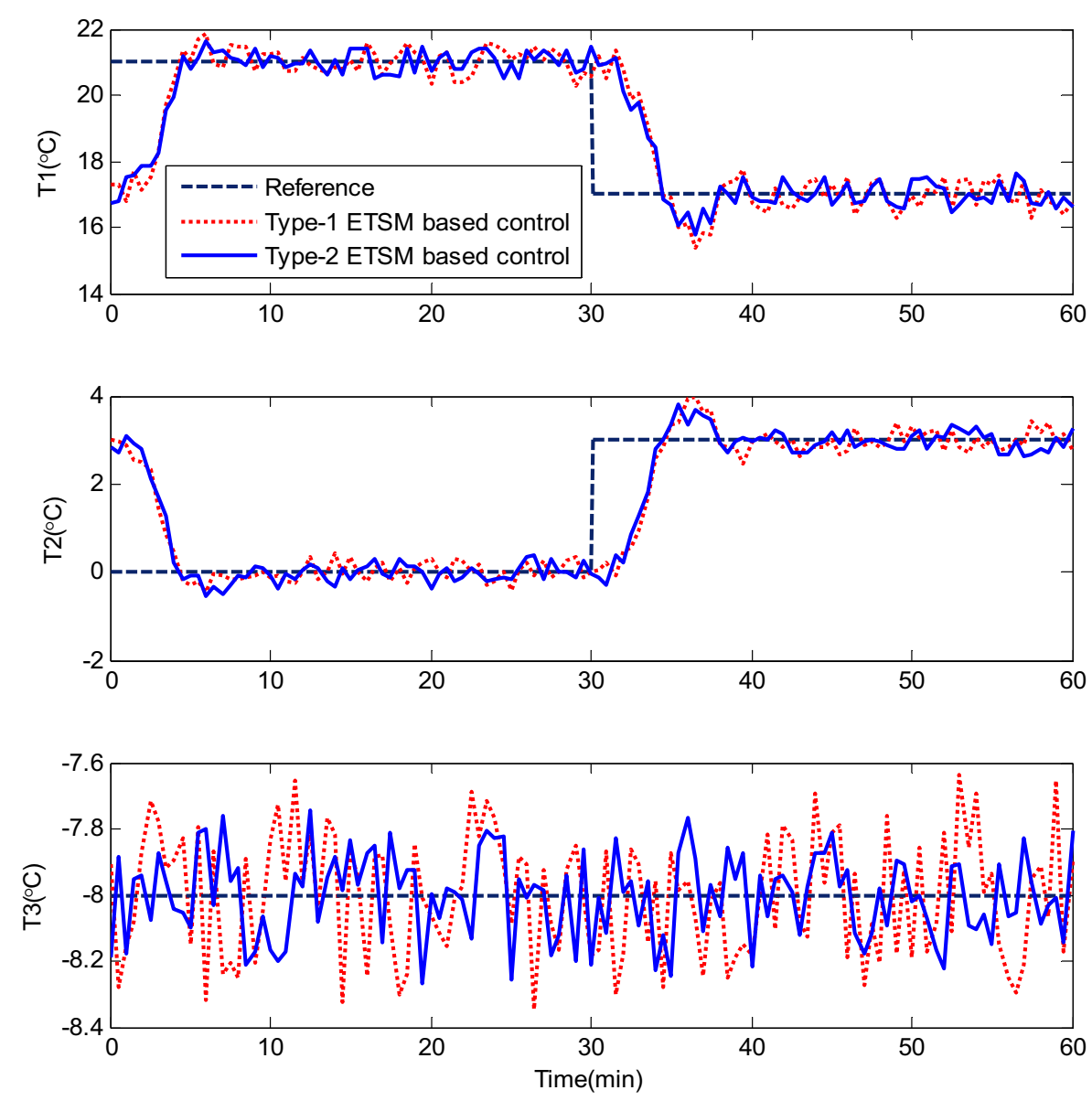

Fig. 10. Type-1 and Type-2 ETSM based decentralized control for the refrigeration system.

Table 4

Performance indexes of Type-1 and Type-2 ETSM based control for the multi-evaporator refrigeration system.

\begin{tabular}{|c|c|c|c|c|c|c|c|c|c|c|c|c|}
\hline & \multicolumn{3}{|l|}{ IAE } & \multicolumn{3}{|l|}{ ISE } & \multicolumn{3}{|l|}{ ITAE } & \multicolumn{3}{|l|}{ ITSE } \\
\hline & $y_{1}$ & $y_{2}$ & $y_{3}$ & $y_{1}$ & $y_{2}$ & $y_{3}$ & $y_{1}$ & $y_{2}$ & $y_{3}$ & $y_{1}$ & $y_{2}$ & $y_{3}$ \\
\hline Type-1 & 38.3 & 25.6 & 9.4 & 62.3 & 34.7 & 1.96 & 973.7 & 609.4 & 280.3 & 1272.2 & 690.6 & 58.2 \\
\hline Type-2 & 34.6 & 25.0 & 6.4 & 52.3 & 34.5 & 0.96 & 850.8 & 575.6 & 184.2 & 1036.7 & 629.2 & 26.7 \\
\hline
\end{tabular}

\section{Conclusions}

This paper presented Type-1 and Type-2 ETSM methods to measure and describe interactions to facilitate decentralized controller design. For each control pair of an MIMO process, a Type-1/Type-2 ETSM can be built by merging the steady and the dynamic interacting effects, quantified by RNGA based criterion, into its individual open-loop Type-1/Type-2 T-S fuzzy model through simply scaling the coefficients. Based on each ETSM, a local controller can be independently devised by using linear SISO control algorithms, and then a decentralized control system can be formed by assembling all these local controllers to manipulate a nonlinear MIMO process. Compared to the existing decentralized fuzzy control methods inserting extra terms in the individual open-loop models to express the interacting effects, ETSM method can greatly reduce the cost and complexity in modeling and controller designs. While compared to the existing RNGA based ETF method, ETSM method can be implemented without requiring exact mathematical process functions and is able to provide more satisfactory control results. Type-2 ETSMs with additional degrees of fuzziness can achieve more robust performances than the Type-1 counterparts under the influence of uncertainties, which have been proved by simulation and experimental results. Since an ETSM can express the interacting effects, more interesting topics, such as block decentralized control and sparse control, can be investigated. These topics will be reported later.

\section{Acknowledgements}

The work was funded by National Research Foundation of Singapore: NRF2011 NRF-CRP001-090. School of Electrical \& Electronic Engineering, Nanyang Technological University is also acknowledged. 


\section{Appendix A.}

The parameters of Type- 1 and Type-2 T-S fuzzy models for the process in Eq. (44) are given in Table A1 and A2, where $R^{l}$ denotes $R u l e$

Table A1

The centers of fuzzy clusters for the process in Eq. (44).

\begin{tabular}{|c|c|c|c|c|c|c|c|}
\hline \multirow{2}{*}{$\begin{array}{l}\text { Centers of } C_{i j}^{l} \text {, } s \\
\text { in loop } y_{i}-u_{j}\end{array}$} & & \multicolumn{6}{|c|}{ No. of fuzzy clusters } \\
\hline & & $\mathrm{R}^{1}(\mathrm{l}=1)$ & $\mathrm{R}^{2}(\mathrm{l}=2)$ & $R^{3}(l=3)$ & $\mathrm{R}^{4}(\mathrm{l}=4)$ & $\mathrm{R}^{5}(\mathrm{l}=5)$ & $\mathrm{R}^{6}(\mathrm{l}=6)$ \\
\hline \multirow{5}{*}{$\begin{array}{l}f_{T S, 11} \\
\left(\Delta \mu_{11}^{l}=0.05\right)\end{array}$} & $u_{c, 1}^{l}-\tau_{11}$ & 0.5622 & 1.3647 & 0.8080 & 1.2917 & 1.3446 & 0.6003 \\
\hline & $y_{c, 1}^{c} 1$ & 1.3286 & 0.9915 & 1.2043 & 1.1904 & 1.5125 & 0.9530 \\
\hline & $y_{c, 1}^{l, 1} 2$ & 1.1450 & 1.1523 & 1.3147 & 1.0916 & 1.3482 & 1.1075 \\
\hline & $y_{c, 1}^{l}$ & 1.1055 & 1.2062 & 1.0991 & 1.3174 & 1.6284 & 0.8485 \\
\hline & $u_{c, 2}^{l} \tau_{12}$ & 1.2909 & 0.8570 & 1.3501 & 0.5789 & 0.6350 & 1.2364 \\
\hline \multirow{3}{*}{$\begin{array}{l}f_{T S, 12} \\
\left(\Delta \mu_{12}^{l}=0.05\right)\end{array}$} & $y^{l}{ }^{l} 1$ & 1.0727 & 0.9838 & 0.9385 & 1.0686 & 0.9503 & 1.0267 \\
\hline & $y_{c, 1}^{l, 1} 2$ & 1.0145 & 1.0085 & 0.9997 & 1.0040 & 1.0149 & 0.9979 \\
\hline & $y_{c, 1}^{l}$ & 1.1222 & 0.9727 & 1.0153 & 0.9847 & 0.8888 & 1.0575 \\
\hline \multirow{4}{*}{$\begin{array}{l}f_{T S, 13} \\
\left(\Delta \mu_{13}^{l}=0.05\right)\end{array}$} & $u_{c, 3}^{l}-\tau_{13}$ & 0.6910 & 0.6976 & 1.2953 & 1.2328 & 1.3997 & 0.6252 \\
\hline & $y^{l}{ }^{l} 1$ & 1.1078 & 1.0586 & 1.0414 & 1.0761 & 1.0703 & 1.0272 \\
\hline & $y_{c, 1}^{l, 1} 2$ & 1.0911 & 1.0471 & 1.0593 & 1.0804 & 1.0460 & 1.0584 \\
\hline & $y_{c, 1}^{l}$ & 1.0822 & 1.0196 & 1.0782 & 1.0774 & 1.1201 & 1.0064 \\
\hline \multirow{4}{*}{$\begin{array}{l}f_{T S, 21} \\
\left(\Delta \mu_{21}^{l}=0.05\right)\end{array}$} & $u_{c, 1}^{l}-\tau_{21}$ & 1.1255 & 0.9491 & 0.5658 & 1.4285 & 0.5358 & 1.3894 \\
\hline & $y_{c, 2^{-}}^{l} 1$ & 2.0363 & 2.1622 & 2.1112 & 2.0109 & 1.9872 & 2.1456 \\
\hline & $y_{c, 2}^{l, 2}-2$ & 1.9934 & 2.2249 & 2.0555 & 2.0733 & 2.0470 & 2.0585 \\
\hline & $y_{c, 2}^{l}$ & 2.0275 & 2.1363 & 2.0055 & 2.1058 & 1.9364 & 2.2626 \\
\hline \multirow{4}{*}{$\begin{array}{l}f_{T S, 22} \\
\left(\Delta \mu_{22}^{l}=0.05\right)\end{array}$} & $u_{c, 2}^{l} \tau_{22}^{c, 2}$ & 1.1127 & 0.8016 & 0.5747 & 1.4044 & 1.2765 & 0.7716 \\
\hline & $y_{c, 2-1}^{l}$ & 0.5326 & 0.4769 & 0.5144 & 0.5023 & 0.4934 & 0.5007 \\
\hline & $y_{c, 2}^{l}-2$ & 0.5303 & 0.4824 & 0.5160 & 0.4970 & 0.5015 & 0.4931 \\
\hline & $y_{c, 2}^{l}$ & 0.5327 & 0.4761 & 0.5152 & 0.5022 & 0.5039 & 0.4902 \\
\hline \multirow{4}{*}{$\begin{array}{l}f_{T S, 23} \\
\left(\triangle \mu_{23}^{l}=0.05\right)\end{array}$} & $u_{c, 3}^{l}-\tau_{23}$ & 1.2047 & 0.7098 & 1.3109 & 0.6307 & 0.8037 & 1.2922 \\
\hline & $y_{c, 2}^{l}-1$ & 0.3152 & 0.2388 & 0.2396 & 0.3261 & 0.2656 & 0.3328 \\
\hline & $y_{c, 2}^{l, 2}-2$ & 0.2842 & 0.2888 & 0.2839 & 0.2673 & 0.2936 & 0.2966 \\
\hline & $y_{c, 2}^{l}$ & 0.3275 & 0.2264 & 0.3076 & 0.2541 & 0.2461 & 0.3571 \\
\hline \multirow{4}{*}{$\begin{array}{l}f_{T S, 31} \\
\left(\Delta \mu_{31}^{l}=0.05\right)\end{array}$} & $u_{c, 1}^{l}-\tau_{31}$ & 1.3618 & 0.6121 & 0.7111 & 1.2980 & 1.3706 & 0.6179 \\
\hline & $y_{c, 3-1}^{l}{ }^{1}$ & 0.2010 & 0.2782 & 0.2186 & 0.2335 & 0.2980 & 0.2037 \\
\hline & $y_{c, 3}^{l, 3} 2$ & 0.2405 & 0.2419 & 0.2171 & 0.2216 & 0.2627 & 0.2448 \\
\hline & $y_{c, 3}^{l}$ & 0.2425 & 0.2354 & 0.1998 & 0.2574 & 0.3258 & 0.1773 \\
\hline \multirow{4}{*}{$\begin{array}{l}f_{T S, 32} \\
\left(\Delta \mu_{32}^{l}=0.05\right)\end{array}$} & $u_{c, 2}^{l}-\tau_{32}$ & 1.3431 & 0.6205 & 1.2799 & 0.6305 & 1.2034 & 0.8962 \\
\hline & $y_{c, 3}^{l}-1$ & 0.6055 & 0.7323 & 0.7242 & 0.6044 & 0.7122 & 0.6536 \\
\hline & $y_{c, 3}^{l, 3}-2$ & 0.6633 & 0.6627 & 0.6880 & 0.6667 & 0.6706 & 0.6782 \\
\hline & $y_{c, 3}^{l}$ & 0.6858 & 0.6514 & 0.7517 & 0.5572 & 0.7462 & 0.6429 \\
\hline \multirow{4}{*}{$\begin{array}{l}f_{T S, 33} \\
\left(\Delta \mu_{33}^{l}=0.03\right)\end{array}$} & $u_{c, 3}^{l} \tau_{33}$ & 0.8772 & 1.2857 & 0.6684 & 1.2590 & 0.5560 & 1.3119 \\
\hline & $y_{c, 3}^{l}-1$ & 0.1077 & 0.1117 & 0.1109 & 0.1092 & 0.1096 & 0.1102 \\
\hline & $y_{c, 3}^{l, 3}-2$ & 0.1080 & 0.1111 & 0.1090 & 0.1113 & 0.1105 & 0.1093 \\
\hline & $y_{c, 3}^{l}$ & 0.1082 & 0.1130 & 0.1086 & 0.1100 & 0.1109 & 0.1086 \\
\hline
\end{tabular}

$l, M_{i j}$ 's $(i, j=1,2,3)$ are chosen as 6.

When $\hat{f}_{T S, i j}$ of control pair $y_{i}-u_{j}$ is a Type- 1 ETSM with $p=0$ and $q=2$, it is:

$$
\begin{array}{rcc}
\text { Rule } & l: \text { IF } & \boldsymbol{x}_{i j}(k) \text { is } C_{i j}^{l} \\
\text { THEN } & y_{i}^{l}(k)=\hat{a}_{i j, 0}^{l} \cdot u_{j}\left(k-\hat{\tau}_{i j}\right)+b_{i j, 1}^{l} \cdot y_{i}(k-1)+b_{i j, 2}^{l} \cdot y_{i}(k-2)
\end{array}
$$

Applying gain and phase margins based control algorithm [15,17] on the linear polynomial of lth fuzzy rule can calculate a control variable $u_{j}^{l}(k)$ by [19]:

$$
u_{j}^{l}(k)=u_{j}(k-1)+\frac{\pi\left(r v_{i}(k)-y_{i}(k)\right)-\pi b_{i j, 1}^{l}\left(r v_{i}(k-1)-y_{i}(k-1)\right)-\pi b_{i j, 2}^{l}\left(r v_{i}(k-2)-y_{i}(k-2)\right)}{2 \hat{a}_{i j, 0}^{l} A_{m, i j} \hat{\tau}_{i j}}
$$

where $A_{m, i j}$ is the gain margin for the control system. According to the requirement, $A_{m, i j}=3$, which associated with a phase margin of $\pi / 3$ $[15,17]$. According to parallel distributed compensation [4], the total control variable $u_{j}(k)$ is a weighted sum of $u_{j}^{l}(k)\left(l=1, \ldots, M_{i j}\right)$ and share the same fuzzy memberships with Eq. (A.1):

$$
u_{j}(k)=\sum_{l=1}^{M_{i j}} \mu_{i j}^{l}\left(\boldsymbol{x}_{i j}(k)\right) u_{j}^{l}(k)
$$


Table A2

The parameters of Type- 1 and Type-2 T-S fuzzy models for the process in Eq. (44).

\begin{tabular}{|c|c|c|c|c|c|c|c|c|c|c|}
\hline \multicolumn{2}{|c|}{ No. of fuzzy rules } & \multicolumn{3}{|c|}{ Type-1 fuzzy model } & \multicolumn{6}{|c|}{ Type-2 fuzzy model } \\
\hline & & $a_{i j, 0}^{l}$ & $b_{i j, 1}^{l}$ & $b_{i j, 2}^{l}$ & $a_{i j, l b, 0}^{l}$ & $b_{i j, l b, 1}^{l}$ & $b_{i j, l b, 2}^{l}$ & $a_{i j, r b, 0}^{l}$ & $b_{i j, r b, 1}^{l}$ & $b_{i j, r b, 2}^{l}$ \\
\hline \multirow{7}{*}{$f_{T S, 11}$} & $\mathrm{R}^{1}(\mathrm{l}=1)$ & 0.4168 & 0.6234 & 0.0503 & 0.4362 & 0.6849 & 0.0205 & 0.3974 & 0.5620 & 0.0801 \\
\hline & $R^{2}(l=2)$ & 0.3746 & 0.8545 & -0.1103 & 0.4063 & 0.8622 & -0.1055 & 0.3429 & 0.8468 & -0.1150 \\
\hline & $\mathrm{R}^{3}(\mathrm{l}=3)$ & 0.3863 & 0.8449 & -0.1765 & 0.4012 & 0.8446 & -0.1448 & 0.3714 & 0.8452 & -0.2082 \\
\hline & $\mathrm{R}^{4}(\mathrm{l}=4)$ & 0.3621 & 0.7834 & -0.1002 & 0.3859 & 0.8059 & -0.0979 & 0.3383 & 0.7608 & -0.1025 \\
\hline & $\mathrm{R}^{5}(\mathrm{l}=5)$ & 0.4159 & 0.7726 & -0.0442 & 0.4317 & 0.8055 & -0.0558 & 0.4001 & 0.7396 & -0.0327 \\
\hline & $\mathrm{R}^{6}(\mathrm{l}=6)$ & 0.3931 & 0.7623 & -0.0971 & 0.4217 & 0.7919 & -0.0774 & 0.3646 & 0.7327 & -0.1169 \\
\hline & $\mathrm{R}^{1}(\mathrm{l}=1)$ & 0.1853 & 0.7450 & 0.0959 & 0.1948 & 0.7958 & 0.0751 & 0.1758 & 0.6943 & 0.1168 \\
\hline \multirow{5}{*}{$f_{T S, 12}$} & $R^{2}(l=2)$ & 0.1745 & 0.6941 & 0.1611 & 0.1825 & 0.7393 & 0.1618 & 0.1665 & 0.6489 & 0.1604 \\
\hline & $\mathrm{R}^{3}(\mathrm{l}=3)$ & 0.1845 & 0.7492 & 0.0625 & 0.1997 & 0.7782 & 0.0625 & 0.1693 & 0.7202 & 0.0626 \\
\hline & $\mathrm{R}^{4}(\mathrm{l}=4)$ & 0.1831 & 0.7475 & 0.0791 & 0.1858 & 0.8249 & 0.0418 & 0.1805 & 0.6702 & 0.1164 \\
\hline & $\mathrm{R}^{5}(\mathrm{l}=5)$ & 0.1519 & 0.6249 & 0.1768 & 0.1594 & 0.6537 & 0.1946 & 0.1443 & 0.5960 & 0.1590 \\
\hline & $\mathrm{R}^{6}(\mathrm{l}=6)$ & 0.1822 & 0.6979 & 0.0894 & 0.1916 & 0.7502 & 0.0701 & 0.1727 & 0.6456 & 0.1086 \\
\hline \multirow{7}{*}{$f_{T S, 13}$} & $\mathrm{R}^{1}(\mathrm{l}=1)$ & 0.0980 & 0.7854 & 0.1420 & 0.0991 & 0.8415 & 0.1272 & 0.0969 & 0.7293 & 0.1569 \\
\hline & $\mathrm{R}^{2}(\mathrm{l}=2)$ & 0.0931 & 0.8095 & 0.0692 & 0.0971 & 0.8475 & 0.0773 & 0.0891 & 0.7716 & 0.0610 \\
\hline & $\mathrm{R}^{3}(\mathrm{l}=3)$ & 0.0946 & 0.8083 & 0.1186 & 0.1047 & 0.8468 & 0.1152 & 0.0845 & 0.7699 & 0.1219 \\
\hline & $\mathrm{R}^{4}(\mathrm{l}=4)$ & 0.0919 & 0.7504 & 0.1254 & 0.0987 & 0.7647 & 0.1474 & 0.0851 & 0.7361 & 0.1034 \\
\hline & $\mathrm{R}^{5}(\mathrm{l}=5)$ & 0.1062 & 0.6681 & 0.2587 & 0.1147 & 0.7141 & 0.2457 & 0.0978 & 0.6222 & 0.2717 \\
\hline & $\mathrm{R}^{6}(\mathrm{l}=6)$ & 0.0956 & 0.8711 & 0.0580 & 0.1010 & 0.9326 & 0.0447 & 0.0902 & 0.8096 & 0.0712 \\
\hline & $\mathrm{R}^{1}(\mathrm{l}=1)$ & 0.1881 & 1.0126 & -0.1523 & 0.2011 & 1.0316 & -0.1280 & 0.1751 & 0.9935 & -0.1766 \\
\hline \multirow{5}{*}{$f_{T S, 21}$} & $\mathrm{R}^{2}(\mathrm{l}=2)$ & 0.2028 & 0.8467 & 0.0421 & 0.2154 & 0.8228 & 0.0992 & 0.1903 & 0.8705 & -0.0151 \\
\hline & $\mathrm{R}^{3}(\mathrm{l}=3)$ & 0.2350 & 1.0616 & -0.1896 & 0.2459 & 1.0841 & -0.1675 & 0.2242 & 1.0391 & -0.2116 \\
\hline & $\mathrm{R}^{4}(\mathrm{l}=4)$ & 0.2127 & 0.8061 & 0.0828 & 0.2404 & 0.8094 & 0.1061 & 0.1851 & 0.8028 & 0.0595 \\
\hline & $\mathrm{R}^{5}(\mathrm{l}=5)$ & 0.2605 & 1.0580 & -0.1312 & 0.2805 & 1.0763 & -0.1038 & 0.2406 & 1.0397 & -0.1586 \\
\hline & $\mathrm{R}^{6}(\mathrm{l}=6)$ & 0.1848 & 0.9333 & 0.0234 & 0.2087 & 0.9642 & 0.0203 & 0.1608 & 0.9024 & 0.0265 \\
\hline \multirow{7}{*}{$f_{T S, 22}$} & $\mathrm{R}^{1}(\mathrm{l}=1)$ & 0.0254 & 1.1676 & -0.2225 & 0.0262 & 1.2035 & -0.2183 & 0.0245 & 1.1316 & -0.2267 \\
\hline & $\mathrm{R}^{2}(\mathrm{l}=2)$ & 0.0159 & 1.2794 & -0.2973 & 0.0166 & 1.3471 & -0.3081 & 0.0151 & 1.2116 & -0.2864 \\
\hline & $\mathrm{R}^{3}(\mathrm{l}=3)$ & 0.0326 & 1.1339 & -0.1470 & 0.0336 & 1.1633 & -0.1308 & 0.0316 & 1.1045 & -0.1632 \\
\hline & $\mathrm{R}^{4}(\mathrm{l}=4)$ & 0.0242 & 1.3626 & -0.4637 & 0.0299 & 1.4041 & -0.4739 & 0.0185 & 1.3212 & -0.4534 \\
\hline & $\mathrm{R}^{5}(\mathrm{l}=5)$ & 0.0173 & 1.4796 & -0.4639 & 0.0218 & 1.5131 & -0.4600 & 0.0128 & 1.4460 & -0.4678 \\
\hline & $\mathrm{R}^{6}(\mathrm{l}=6)$ & 0.0140 & 1.4328 & -0.5218 & 0.0169 & 1.4874 & -0.5301 & 0.0112 & 1.3782 & -0.5134 \\
\hline & $\mathrm{R}^{1}(\mathrm{l}=1)$ & 0.1431 & 0.4784 & 0.0008 & 0.1470 & 0.5093 & -0.0062 & 0.1393 & 0.4475 & 0.0078 \\
\hline \multirow{5}{*}{$f_{T S, 23}$} & $\mathrm{R}^{2}(\mathrm{l}=2)$ & 0.1472 & 0.5180 & 0.0102 & 0.1520 & 0.5509 & 0.0226 & 0.1424 & 0.4850 & -0.0023 \\
\hline & $\mathrm{R}^{3}(\mathrm{l}=3)$ & 0.1460 & 0.4818 & -0.0028 & 0.1525 & 0.4978 & -0.0001 & 0.1395 & 0.4657 & -0.0055 \\
\hline & $\mathrm{R}^{4}(\mathrm{l}=4)$ & 0.1448 & 0.4781 & 0.0295 & 0.1475 & 0.5224 & 0.0189 & 0.1422 & 0.4337 & 0.0400 \\
\hline & $\mathrm{R}^{5}(\mathrm{l}=5)$ & 0.1413 & 0.4808 & -0.0026 & 0.1466 & 0.5059 & 0.0115 & 0.1360 & 0.4557 & -0.0167 \\
\hline & $\mathrm{R}^{6}(\mathrm{l}=6)$ & 0.1495 & 0.5094 & -0.0118 & 0.1539 & 0.5300 & -0.0112 & 0.1451 & 0.4889 & -0.0124 \\
\hline \multirow{7}{*}{$f_{T S, 31}$} & $\mathrm{R}^{1}(\mathrm{l}=1)$ & 0.0788 & 0.8838 & -0.1595 & 0.0849 & 0.8820 & -0.1458 & 0.0727 & 0.8857 & -0.1731 \\
\hline & $\mathrm{R}^{2}(\mathrm{l}=2)$ & 0.0852 & 0.6130 & 0.0631 & 0.0864 & 0.6812 & 0.0276 & 0.0839 & 0.5447 & 0.0985 \\
\hline & $\mathrm{R}^{3}(\mathrm{l}=3)$ & 0.0823 & 0.6856 & -0.0409 & 0.0855 & 0.7638 & -0.0673 & 0.0792 & 0.6073 & -0.0145 \\
\hline & $\mathrm{R}^{4}(\mathrm{l}=4)$ & 0.0747 & 0.7549 & -0.0992 & 0.0796 & 0.7771 & -0.0971 & 0.0698 & 0.7328 & -0.1014 \\
\hline & $\mathrm{R}^{5}(\mathrm{l}=5)$ & 0.0802 & 0.7467 & -0.0008 & 0.0841 & 0.7766 & -0.0119 & 0.0763 & 0.7168 & 0.0103 \\
\hline & $\mathrm{R}^{6}(\mathrm{l}=6)$ & 0.0818 & 0.8178 & -0.1564 & 0.0864 & 0.7850 & -0.0949 & 0.0773 & 0.8506 & -0.2180 \\
\hline & $\mathrm{R}^{1}(\mathrm{l}=1)$ & 0.1778 & 0.6767 & 0.0550 & 0.1896 & 0.7009 & 0.0570 & 0.1661 & 0.6526 & 0.0530 \\
\hline \multirow{5}{*}{$f_{T S, 32}$} & $\mathrm{R}^{2}(\mathrm{l}=2)$ & 0.1789 & 0.6433 & 0.1093 & 0.1830 & 0.7056 & 0.0848 & 0.1748 & 0.5811 & 0.1338 \\
\hline & $\mathrm{R}^{3}(\mathrm{l}=3)$ & 0.1711 & 0.6716 & 0.0518 & 0.1784 & 0.7101 & 0.0411 & 0.1639 & 0.6331 & 0.0624 \\
\hline & $\mathrm{R}^{4}(\mathrm{l}=4)$ & 0.1670 & 0.6305 & 0.0966 & 0.1762 & 0.6755 & 0.1000 & 0.1579 & 0.5854 & 0.0932 \\
\hline & $\mathrm{R}^{5}(\mathrm{l}=5)$ & 0.1775 & 0.7149 & 0.0543 & 0.1857 & 0.7500 & 0.0474 & 0.1693 & 0.6797 & 0.0613 \\
\hline & $\mathrm{R}^{6}(\mathrm{l}=6)$ & 0.1799 & 0.6413 & 0.0997 & 0.1944 & 0.6931 & 0.0796 & 0.1654 & 0.5895 & 0.1198 \\
\hline \multirow{6}{*}{$f_{T S, 33}$} & $\mathrm{R}^{1}(\mathrm{l}=1)$ & 0.0024 & 1.0770 & -0.0830 & 0.0029 & 1.1021 & -0.0621 & 0.0019 & 1.0520 & -0.1040 \\
\hline & $\mathrm{R}^{2}(\mathrm{l}=2)$ & 0.0038 & 0.6604 & 0.3255 & 0.0045 & 0.6959 & 0.3242 & 0.0030 & 0.6248 & 0.3268 \\
\hline & $\mathrm{R}^{3}(\mathrm{l}=3)$ & 0.0005 & 1.0561 & -0.1007 & 0.0007 & 1.0956 & -0.0965 & 0.0003 & 1.0166 & -0.1050 \\
\hline & $\mathrm{R}^{4}(\mathrm{l}=4)$ & 0.0035 & 0.6166 & 0.3367 & 0.0041 & 0.5863 & 0.4023 & 0.0029 & 0.6469 & 0.2711 \\
\hline & $\mathrm{R}^{5}(\mathrm{l}=5)$ & 0.0027 & 0.9986 & 0.0155 & 0.0029 & 1.0034 & 0.0547 & 0.0025 & 0.9938 & -0.0236 \\
\hline & $\mathrm{R}^{6}(\mathrm{l}=6)$ & 0.0029 & 0.8095 & 0.1177 & 0.0035 & 0.8531 & 0.1108 & 0.0023 & 0.7660 & 0.1246 \\
\hline
\end{tabular}

When $\hat{f}_{T S, i j}$ is a Type-2 T-S fuzzy model with $p=0$ and $q=2$, it is:

$\begin{array}{llc}\text { Rule } & l: \text { IF } & \boldsymbol{x}_{i j}(k) \text { is } \tilde{C}_{i j}^{l} \\ & \text { THEN } & \tilde{y}_{i}^{l}(k)=\hat{\tilde{a}}_{i j, 0}^{l} \cdot u_{j}\left(k-\hat{\tau}_{i j}\right)+\tilde{b}_{i j, 1}^{l} \cdot y_{i}(k-1)+\cdots+\tilde{b}_{i j, 2}^{l} \cdot y_{i}(k-2)\end{array}$

where $\tilde{y}_{i}^{l}(k)=\left[y_{i, l b}^{l}(k), \quad y_{i, r b}^{l}(k)\right]$ that

$$
\left\{\begin{array}{l}
y_{i, l b}^{l}(k)=\hat{a}_{i j, l b, 0}^{l} \cdot u_{j}\left(k-\hat{\tau}_{i j}\right)+b_{i j, l b, 1}^{l} y_{i}(k-1)+b_{i j, l b, 2}^{l} \cdot y_{i}(k-2) \\
y_{i, r b}^{l}(k)=\hat{a}_{i j, r b, 0}^{l} \cdot u_{j}\left(k-\hat{\tau}_{i j}\right)+b_{i j, r b, 1}^{l} y_{i}(k-1)+b_{i j, r b, 2}^{l} \cdot y_{i}(k-2)
\end{array}\right.
$$

Based on the two linear polynomials in Eq. (A.4), two control variables, denoted by $u_{j, l b}^{l}(k)$ and $u_{j . r b}^{l}(k)$, can be calculated using the gain and phase margins based control algorithm. Then a control variable interval $\tilde{u}_{j}(k)$ [25] can be obtained by: 


$$
\tilde{u}_{j}(k)=\left[u_{j, l b}(k), \quad u_{j, r b}(k)\right]=\left[\frac{\sum_{l=1}^{M_{i j}} \mu_{i j, l b}^{l}\left(\boldsymbol{x}_{i j}(k)\right) u_{j, l b}^{l}(k)}{\sum_{l=1}^{M_{i j}} \mu_{i j, l b}^{l}\left(\boldsymbol{x}_{i j}(k)\right)}, \frac{\sum_{l=1}^{M_{i j}} \mu_{i j, r b}^{l}\left(\boldsymbol{x}_{i j}(k)\right) u_{j, r b}^{l}(k)}{\sum_{l=1}^{M_{i i}} \mu_{i j, r b}^{l}\left(\boldsymbol{x}_{i j}(k)\right)}\right]
$$

The total control variable $u_{j}(k)$ is derived by defuzzifying $\tilde{u}_{j}(k)[25]$ as

$$
u_{j}(k)=\frac{u_{j, l b}(k)+u_{j, r b}(k)}{2}
$$

Linearize the process in Eq. (44) at the given operating points to have the following transfer function matrix:

$$
\boldsymbol{G}(s)=\left[g_{i j}(s)\right]_{n \times n}=\left[\begin{array}{ccc}
\frac{1.25}{0.25 s+1} e^{-2 s} & \frac{1}{0.5 s+1} e^{-2 s} & \frac{1}{s+1} e^{-s} \\
\frac{2}{s+1} e^{-2 s} & \frac{0.5}{0.1667 s^{2}+0.8333 s+1} e^{-2 s} & \frac{0.2857}{0.1429 s+1} e^{-s} \\
\frac{0.25}{0.25 s+1} e^{-2 s} & \frac{0.6667}{0.3333 s+1} e^{-2 s} & \frac{0.1}{0.5 s^{2}+1.5 s+1} e^{-s}
\end{array}\right]
$$

Loop pairing structure selected using RNGA based criterion is $y_{1}-u_{3} / y_{2}-u_{1} / y_{3}-u_{2}$. The ETFs $\hat{g}_{i j}(s)$ 's and the controllers $G_{c, i}(s)(i=1$, 2 , 3 ) designed with the required gain and phase margins which are 3 and $\pi / 3$ are:

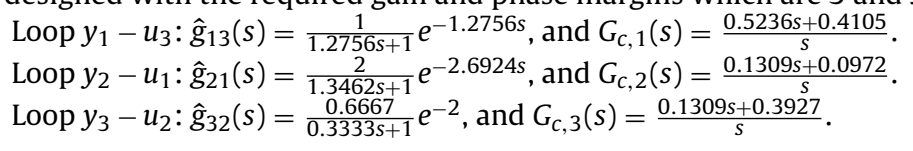

\section{Appendix B.}

The parameters of Type-1 and Type-2 T-S fuzzy models for the multi-evaporator refrigeration system are given in Table B1 and B2, where $R^{l}$ denotes Rule $\left.l, M_{i j}=6, i, j=1,2,3\right)$.

\begin{tabular}{|c|c|c|c|c|c|c|c|}
\hline \multirow{2}{*}{\multicolumn{2}{|c|}{ Centers of $C_{i j}^{l}$ 's in loop $y_{i}-u_{j}$}} & \multicolumn{6}{|c|}{ No. of fuzzy clusters } \\
\hline & & $\mathrm{R}^{1}(\mathrm{l}=1)$ & $\mathrm{R}^{2}(\mathrm{l}=2)$ & $\mathrm{R}^{3}(\mathrm{l}=3)$ & $\mathrm{R}^{4}(\mathrm{l}=4)$ & $\mathrm{R}^{5}(\mathrm{l}=5)$ & $\mathrm{R}^{6}(\mathrm{l}=6)$ \\
\hline \multirow{4}{*}{$\begin{array}{l}f_{T S, 11} \\
\left(\triangle \mu_{11}^{l}=0.05\right)\end{array}$} & $u^{l}{ }^{1}-\tau_{11}$ & -0.0895 & -0.0753 & -0.0958 & -0.0379 & -0.0614 & -0.1178 \\
\hline & $y_{c, 1}^{l}-1$ & 0.1123 & 0.1214 & 0.0602 & 0.2745 & 0.2665 & 0.0027 \\
\hline & $y_{c, 1}^{l} 2$ & 0.0469 & 0.2542 & 0.1135 & 0.1654 & 0.0066 & 0.2075 \\
\hline & $y_{c, 1}^{l}$ & 0.1584 & 0.1340 & 0.1632 & 0.0865 & 0.1211 & 0.1965 \\
\hline \multirow{4}{*}{$\begin{array}{l}f_{T S, 12} \\
\left(\Delta \mu_{12}^{l}=0.05\right)\end{array}$} & $u_{c, 2}^{l}-\tau_{12}$ & -1.2258 & 0.4658 & -0.6004 & 0.7367 & -0.6717 & 0.8497 \\
\hline & $y_{c, 1}^{l}-1$ & -0.5747 & 0.2136 & 0.2547 & -0.1123 & 0.3494 & -0.4642 \\
\hline & $y_{c, 1}^{l,} 2$ & -0.0492 & -0.3563 & 0.1002 & 0.2441 & 0.1283 & -0.3066 \\
\hline & $y_{c, 1}^{l}$ & -0.6855 & 0.2591 & -0.1491 & 0.2547 & -0.1602 & 0.1935 \\
\hline \multirow{4}{*}{$\begin{array}{l}f_{T S, 13} \\
\left(\Delta \mu_{13}^{l}=0.03\right)\end{array}$} & $u_{c, 3-}^{l} \tau_{13}$ & -0.7445 & 0.8525 & -1.0112 & 0.3896 & 0.8216 & -0.8990 \\
\hline & $y_{c, 1}^{l}-1$ & 0.1154 & -0.1251 & -0.2065 & 0.0716 & 0.0239 & 0.0259 \\
\hline & $y_{c, 1}^{l}-2$ & 0.0342 & 0.0011 & -0.0611 & -0.1126 & 0.0145 & 0.0301 \\
\hline & $y_{c, 1}^{l}$ & -0.0589 & 0.0698 & -0.2045 & 0.0768 & 0.1141 & -0.1077 \\
\hline \multirow{4}{*}{$\begin{array}{l}f_{T S, 21} \\
\left(\Delta \mu_{21}^{l}=0.05\right)\end{array}$} & $u_{c, 1}^{l}-\tau_{21}$ & -0.1187 & -0.0572 & -0.1087 & -0.0453 & -0.0830 & -0.0632 \\
\hline & $y_{c, 2^{-1}}^{l}-1$ & -0.0307 & -0.1017 & -0.0422 & -0.0838 & -0.0611 & -0.0490 \\
\hline & $y_{c, 2}^{l, 2} 2$ & -0.0365 & -0.0673 & -0.0680 & -0.0661 & -0.1000 & -0.0122 \\
\hline & $y_{c, 2}^{l}$ & -0.0823 & $\cdot-0.0565$ & -0.0786 & -0.0449 & -0.0653 & -0.0502 \\
\hline \multirow{4}{*}{$\begin{array}{l}f_{T S, 22} \\
\left(\Delta \mu_{22}^{l}=0.05\right)\end{array}$} & $u_{c, 2}^{l} \tau_{22}$ & -0.0990 & -0.0686 & -0.1687 & 0.0210 & -0.0938 & -0.0671 \\
\hline & $y_{c, 2}^{l}-1$ & 0.0301 & 0.1051 & 0.0699 & 0.0324 & 0.0323 & 0.0510 \\
\hline & $y_{c, 2}^{l, 2} 2$ & 0.0287 & 0.0783 & 0.0851 & -0.0026 & 0.0421 & 0.0729 \\
\hline & $y_{c, 2}^{l}$ & 0.0615 & 0.0588 & 0.1090 & -0.0065 & 0.0589 & 0.0477 \\
\hline \multirow{4}{*}{$\begin{array}{l}f_{T S, 23} \\
\left(\Delta \mu_{23}^{l}=0.03\right)\end{array}$} & $u_{c, 3}^{l}-\tau_{23}$ & 0.0016 & -0.1283 & -0.1004 & -0.0738 & -0.1648 & -0.0101 \\
\hline & $y_{c, 2}^{l}-1$ & -0.0108 & -0.0053 & -0.0030 & -0.0115 & -0.0073 & -0.0075 \\
\hline & $y_{c, 2}^{l, 2}-2$ & -0.0086 & -0.0086 & -0.0094 & -0.0052 & -0.0033 & -0.0083 \\
\hline & $y_{c, 2}^{l}$ & -0.0034 & -0.0103 & -0.0078 & -0.0087 & -0.0136 & -0.0030 \\
\hline \multirow{4}{*}{$\begin{array}{l}f_{T S, 31} \\
\left(\Delta \mu_{31}^{l}=0.03\right)\end{array}$} & $u_{c, 1}^{l}-\tau_{31}$ & -0.0210 & -0.1501 & -0.1141 & -0.0507 & -0.0651 & -0.0758 \\
\hline & $y_{c, 3}^{l}-1$ & -0.0058 & -0.0259 & -0.0092 & -0.0208 & -0.0268 & -0.0039 \\
\hline & $y_{c, 3}^{l, 3} 2$ & -0.0156 & -0.0110 & -0.0052 & -0.0257 & -0.0141 & -0.0163 \\
\hline & $y_{c, 3}^{l}$ & -0.0046 & -0.0291 & -0.0193 & -0.0130 & -0.0167 & -0.0123 \\
\hline \multirow{4}{*}{$\begin{array}{l}f_{T S, 32} \\
\left(\triangle \mu_{32}^{l}=0.05\right)\end{array}$} & $u_{c, 2}^{l}-\tau_{32}$ & -0.0602 & -0.1107 & -0.0780 & -0.1081 & -0.0713 & -0.0484 \\
\hline & $y_{c, 3}^{l}-1$ & -0.0037 & -0.0127 & -0.0134 & -0.0020 & -0.0092 & -0.0052 \\
\hline & $y_{3,3}^{l} 2$ & -0.0050 & -0.0064 & -0.0096 & -0.0064 & -0.0082 & -0.0083 \\
\hline & $y_{c, 3}^{l}$ & -0.0053 & -0.0116 & -0.0094 & -0.0083 & -0.0078 & -0.0050 \\
\hline \multirow{4}{*}{$\begin{array}{l}f_{T S, 33} \\
\left(\Delta \mu_{33}^{l}=0.05\right)\end{array}$} & $u_{c, 3}^{l}-\tau_{33}$ & 0.4512 & 0.5290 & 0.1056 & -1.2862 & -0.9391 & 0.8002 \\
\hline & $y_{c, 3}^{l}-1$ & -0.1045 & 0.1306 & -0.1123 & 0.1961 & -0.1869 & 0.2330 \\
\hline & $y_{c, 3}^{l} 2$ & -0.0183 & -0.1162 & 0.0914 & 0.0075 & -0.0369 & 0.2063 \\
\hline & $y_{c, 3}^{l}$ & -0.1251 & -0.0543 & -0.0580 & 0.3157 & 0.1087 & -0.0659 \\
\hline
\end{tabular}

Table B1

The centers of fuzzy clusters for the multi-evaporator refrigeration system. 
Table B2

The parameters of Type-1 and Type-2 T-S fuzzy models for the multi-evaporator refrigeration system.

\begin{tabular}{|c|c|c|c|c|c|c|c|c|c|c|}
\hline \multicolumn{2}{|c|}{ No. of fuzzy rules } & \multicolumn{3}{|c|}{ Type- 1 fuzzy model } & \multicolumn{6}{|c|}{ Type-2 fuzzy model } \\
\hline & & $a_{i j, 0}^{l}$ & $b_{i j, 1}^{l}$ & $b_{i j, 2}^{l}$ & $a_{i j, l b, 0}^{l}$ & $b_{i j, l b, 1}^{l}$ & $b_{i j, l b, 2}^{l}$ & $a_{i j, r b, 0}^{l}$ & $b_{i j, r b, 1}^{l}$ & $b_{i j, r b, 2}^{l}$ \\
\hline \multirow{6}{*}{$f_{T S, 11}$} & $\mathrm{R}^{1}(\mathrm{l}=1)$ & -1.6533 & 0.0820 & -0.0095 & -1.6535 & 0.0802 & -0.0258 & -1.6532 & 0.0838 & 0.0067 \\
\hline & $\mathrm{R}^{2}(\mathrm{l}=2)$ & -1.6519 & 0.0853 & -0.0080 & -1.6493 & 0.0817 & 0.0078 & -1.6544 & 0.0890 & -0.0239 \\
\hline & $\mathrm{R}^{3}(\mathrm{l}=3)$ & -1.6493 & 0.0938 & -0.0076 & -1.6547 & 0.0781 & -0.0059 & -1.6438 & 0.1094 & -0.0094 \\
\hline & $\mathrm{R}^{4}(\mathrm{l}=4)$ & -1.6452 & 0.0879 & 0.0000 & -1.6336 & 0.1009 & 0.0035 & -1.6568 & 0.0750 & -0.0034 \\
\hline & $R^{5}(l=5)$ & -1.6524 & 0.0771 & 0.0045 & -1.6531 & 0.0881 & -0.0071 & -1.6516 & 0.0661 & 0.0161 \\
\hline & $R^{6}(l=6)$ & -1.6556 & 0.0799 & 0.0037 & -1.6663 & 0.0690 & 0.0111 & -1.6448 & 0.0908 & -0.0037 \\
\hline \multirow{6}{*}{$f_{T S, 12}$} & $\mathrm{R}^{1}(\mathrm{l}=1)$ & 0.4039 & 0.3290 & 0.0022 & 0.3989 & 0.3158 & 0.0048 & 0.4089 & 0.3421 & -0.0004 \\
\hline & $R^{2}(1=2)$ & 0.4027 & 0.3320 & 0.0004 & 0.4049 & 0.3453 & -0.0148 & 0.4005 & 0.3188 & 0.0157 \\
\hline & $\mathrm{R}^{3}(\mathrm{l}=3)$ & 0.3997 & 0.3389 & 0.0026 & 0.3944 & 0.3540 & 0.0065 & 0.4049 & 0.3238 & -0.0013 \\
\hline & $\mathrm{R}^{4}(\mathrm{l}=4)$ & 0.3990 & 0.3316 & -0.0031 & 0.4060 & 0.3251 & 0.0088 & 0.3921 & 0.3382 & -0.0149 \\
\hline & $R^{5}(l=5)$ & 0.4039 & 0.3199 & 0.0015 & 0.3995 & 0.3367 & 0.0014 & 0.4082 & 0.3031 & 0.0016 \\
\hline & $R^{6}(l=6)$ & 0.4041 & 0.3201 & 0.0084 & 0.4085 & 0.3053 & 0.0091 & 0.3997 & 0.3350 & 0.0077 \\
\hline \multirow{6}{*}{$f_{T S, 13}$} & $\mathrm{R}^{1}(\mathrm{l}=1)$ & 0.1320 & 0.3409 & 0.0036 & 0.1302 & 0.3556 & 0.0023 & 0.1338 & 0.3262 & 0.0049 \\
\hline & $R^{2}(l=2)$ & 0.1314 & 0.3385 & 0.0061 & 0.1330 & 0.3227 & 0.0145 & 0.1297 & 0.3543 & -0.0023 \\
\hline & $R^{3}(l=3)$ & 0.1310 & 0.3499 & -0.0043 & 0.1298 & 0.3337 & -0.0018 & 0.1322 & 0.3662 & -0.0067 \\
\hline & $\mathrm{R}^{4}(\mathrm{l}=4)$ & 0.1311 & 0.3560 & -0.0033 & 0.1318 & 0.3738 & -0.0182 & 0.1303 & 0.3382 & 0.0115 \\
\hline & $R^{5}(l=5)$ & 0.1290 & 0.3481 & 0.0011 & 0.1324 & 0.3479 & 0.0026 & 0.1257 & 0.3483 & -0.0004 \\
\hline & $R^{6}(l=6)$ & 0.1296 & 0.3487 & 0.0014 & 0.1269 & 0.3518 & 0.0062 & 0.1323 & 0.3456 & -0.0033 \\
\hline \multirow{6}{*}{$f_{T S, 21}$} & $\mathrm{R}^{1}(\mathrm{l}=1)$ & 0.6465 & 0.1952 & 0.0020 & 0.6395 & 0.2080 & 0.0053 & 0.6535 & 0.1825 & -0.0014 \\
\hline & $R^{2}(l=2)$ & 0.6488 & 0.1974 & -0.0035 & 0.6495 & 0.1793 & -0.0013 & 0.6480 & 0.2155 & -0.0057 \\
\hline & $R^{3}(l=3)$ & 0.6528 & 0.1782 & 0.0012 & 0.6466 & 0.1939 & -0.0008 & 0.6590 & 0.1625 & 0.0033 \\
\hline & $\mathrm{R}^{4}(\mathrm{l}=4)$ & 0.6527 & 0.1831 & -0.0025 & 0.6608 & 0.1723 & -0.0040 & 0.6445 & 0.1939 & -0.0010 \\
\hline & $\mathrm{R}^{5}(\mathrm{l}=5)$ & 0.6459 & 0.1876 & 0.0000 & 0.6467 & 0.1887 & -0.0173 & 0.6452 & 0.1865 & 0.0174 \\
\hline & $R^{6}(l=6)$ & 0.6500 & 0.1862 & -0.0025 & 0.6522 & 0.1863 & 0.0149 & 0.6477 & 0.1860 & -0.0198 \\
\hline \multirow{7}{*}{$f_{T S, 22}$} & $\mathrm{R}^{1}(\mathrm{l}=1)$ & -0.5602 & 0.1823 & -0.0073 & -0.5670 & 0.1766 & -0.0146 & -0.5535 & 0.1880 & -0.0001 \\
\hline & $\mathrm{R}^{2}(\mathrm{l}=2)$ & -0.5644 & 0.1923 & -0.0049 & -0.5617 & 0.2089 & -0.0049 & -0.5670 & 0.1757 & -0.0049 \\
\hline & $\mathrm{R}^{3}(\mathrm{l}=3)$ & -0.5698 & 0.1884 & -0.0020 & -0.5795 & 0.1879 & 0.0024 & -0.5601 & 0.1888 & -0.0064 \\
\hline & $\mathrm{R}^{4}(\mathrm{l}=4)$ & -0.5695 & 0.1889 & 0.0006 & -0.5616 & 0.1867 & -0.0104 & -0.5774 & 0.1910 & 0.0115 \\
\hline & $R^{5}(l=5)$ & -0.5596 & 0.1876 & -0.0147 & -0.5692 & 0.1809 & -0.0002 & -0.5500 & 0.1942 & -0.0291 \\
\hline & $R^{6}(l=6)$ & -0.5622 & 0.1832 & -0.0062 & -0.5584 & 0.1739 & 0.0037 & -0.5659 & 0.1925 & -0.0161 \\
\hline & $\mathrm{R}^{1}(\mathrm{l}=1)$ & 0.0680 & 0.3132 & -0.0002 & 0.0694 & 0.3083 & -0.0028 & 0.0665 & 0.3180 & 0.0025 \\
\hline \multirow{5}{*}{$f_{T S, 23}$} & $R^{2}(l=2)$ & 0.0681 & 0.3154 & -0.0022 & 0.0672 & 0.3203 & -0.0062 & 0.0691 & 0.3105 & 0.0017 \\
\hline & $R^{3}(l=3)$ & 0.0692 & 0.3139 & -0.0004 & 0.0692 & 0.3319 & -0.0124 & 0.0692 & 0.2958 & 0.0115 \\
\hline & $\mathrm{R}^{4}(\mathrm{l}=4)$ & 0.0695 & 0.3079 & -0.0021 & 0.0695 & 0.2891 & 0.0082 & 0.0695 & 0.3268 & -0.0125 \\
\hline & $R^{5}(l=5)$ & 0.0692 & 0.3069 & 0.0017 & 0.0678 & 0.3086 & 0.0070 & 0.0706 & 0.3051 & -0.0035 \\
\hline & $R^{6}(l=6)$ & 0.0694 & 0.3063 & 0.0010 & 0.0709 & 0.3057 & 0.0012 & 0.0680 & 0.3068 & 0.0007 \\
\hline \multirow{6}{*}{$f_{T S, 31}$} & $\mathrm{R}^{1}(\mathrm{l}=1)$ & 0.1483 & 0.2658 & -0.0011 & 0.1507 & 0.2741 & -0.0004 & 0.1458 & 0.2575 & -0.0018 \\
\hline & $\mathrm{R}^{2}(\mathrm{l}=2)$ & 0.1484 & 0.2586 & -0.0042 & 0.1465 & 0.2474 & 0.0018 & 0.1503 & 0.2699 & -0.0101 \\
\hline & $\mathrm{R}^{3}(\mathrm{l}=3)$ & 0.1469 & 0.2476 & 0.0048 & 0.1459 & 0.2500 & 0.0207 & 0.1479 & 0.2452 & -0.0111 \\
\hline & $\mathrm{R}^{4}(\mathrm{l}=4)$ & 0.1482 & 0.2576 & 0.0045 & 0.1488 & 0.2572 & -0.0120 & 0.1477 & 0.2581 & 0.0210 \\
\hline & $R^{5}(l=5)$ & 0.1473 & 0.2501 & 0.0015 & 0.1479 & 0.2326 & 0.0082 & 0.1467 & 0.2676 & -0.0052 \\
\hline & $R^{6}(l=6)$ & 0.1472 & 0.2533 & -0.0029 & 0.1466 & 0.2697 & -0.0118 & 0.1478 & 0.2368 & 0.0061 \\
\hline \multirow{6}{*}{$f_{T S, 32}$} & $\mathrm{R}^{1}(\mathrm{l}=1)$ & 0.0720 & 0.2853 & 0.0049 & 0.0730 & 0.2886 & 0.0162 & 0.0709 & 0.2821 & -0.0065 \\
\hline & $\mathrm{R}^{2}(\mathrm{l}=2)$ & 0.0712 & 0.2942 & -0.0043 & 0.0709 & 0.2769 & 0.0076 & 0.0715 & 0.3114 & -0.0161 \\
\hline & $R^{3}(l=3)$ & 0.0711 & 0.2820 & 0.0054 & 0.0711 & 0.2689 & -0.0018 & 0.0711 & 0.2950 & 0.0125 \\
\hline & $\mathrm{R}^{4}(\mathrm{l}=4)$ & 0.0711 & 0.2843 & 0.0003 & 0.0702 & 0.2974 & -0.0040 & 0.0720 & 0.2712 & 0.0046 \\
\hline & $R^{5}(l=5)$ & 0.0710 & 0.2724 & -0.0065 & 0.0715 & 0.2585 & -0.0061 & 0.0706 & 0.2863 & -0.0069 \\
\hline & $\mathrm{R}^{6}(\mathrm{l}=6)$ & 0.0713 & 0.2739 & 0.0032 & 0.0720 & 0.2912 & -0.0058 & 0.0707 & 0.2567 & 0.0121 \\
\hline \multirow{6}{*}{$f_{T S, 33}$} & $\mathrm{R}^{1}(\mathrm{l}=1)$ & -0.1908 & 0.3747 & -0.0064 & -0.1883 & 0.3584 & -0.0013 & -0.1933 & 0.3911 & -0.0115 \\
\hline & $\mathrm{R}^{2}(\mathrm{l}=2)$ & -0.1898 & 0.3612 & 0.0016 & -0.1882 & 0.3757 & -0.0145 & -0.1914 & 0.3468 & 0.0177 \\
\hline & $\mathrm{R}^{3}(\mathrm{l}=3)$ & -0.1894 & 0.3512 & 0.0061 & -0.1887 & 0.3265 & 0.0188 & -0.1901 & 0.3758 & -0.0066 \\
\hline & $\mathrm{R}^{4}(\mathrm{l}=4)$ & -0.1897 & 0.3667 & -0.0008 & -0.1931 & 0.3767 & -0.0030 & -0.1864 & 0.3567 & 0.0015 \\
\hline & $R^{5}(l=5)$ & -0.1896 & 0.3704 & -0.0032 & -0.1924 & 0.3569 & -0.0014 & -0.1867 & 0.3840 & -0.0050 \\
\hline & $R^{6}(l=6)$ & -0.1895 & 0.3747 & -0.0056 & -0.1879 & 0.3840 & 0.0031 & -0.1911 & 0.3654 & -0.0143 \\
\hline
\end{tabular}

\section{References}

[1] L.X. Wang, J.M. Mendel, Fuzzy basis functions, universal approximation, and orthogonal least-squares learning, IEEE Trans. Neural Netw. 3 (1992) 807-814.

[2] Y. Hao, General SISO Takagi-Sugeno fuzzy systems with linear rule consequent are universal approximators, IEEE Trans. Fuzzy Syst. 6 (1998) $582-587$.

[3] G. Feng, A survey on analysis and design of model-Based fuzzy control systems, IEEE Trans. Fuzzy Syst. 14 (2006) 676-697.

[4] H.O. Wang, K. Tanaka, M.F. Griffin, An approach to fuzzy control of nonlinear systems: stability and design issues, IEEE Trans. Fuzzy Syst. 4 (1996) 14-23.

[5] C.C. Hua, S.X. Ding, Decentralized networked control system design using T-S fuzzy approach, IEEE Trans. Fuzzy Syst. 20 (2012) 9-21.

[6] S.W. Lin, C.H. Sun, C.H. Chiu, Decentralized guaranteed cost control for large-scale T-S fuzzy systems, Int. J. Fuzzy Syst. 12 (2010) $300-310$.

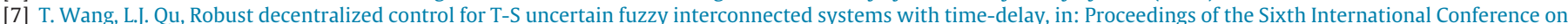
Machine Learning and Cybernetics, Hong Kong, China, 2007, pp. 631-636.

[8] T. Wang, S.C. Tong, Decentralized fuzzy model reference $\mathrm{H} \infty$ tracking control for nonlinear large-scale systems, in: Proceedings of the World Congress on Intelligent Control and Automation, Dalian, China, 2006, pp. 75-79.

[9] H.P. Huang, J.C. Jeng, C.H. Chiang, W. Pan, A direct method for multi-loop PI/PID controller design, J. Process Control 13 (2003) $769-786$.

[10] E.H. Bristol, On a new measure of interaction for multi-variable process control, IEEE Trans. Autom. Control 11 (1966) $133-134$.

[11] E.H. Bristol, Recent results on interactions in multivariable process control, in: Proceedings of the 71 St Annual AIChE Meeting, Houston, TX, USA, 1979.

[12] M. Witcher, T.J. McAvoy, Interacting control systems: steady state and dynamic measurement of interaction, ISA Trans. 16 (1977) 83-90.

[13] L. Tung, T. Edgar, Analysis of control output interactions in dynamic systems, AIChE J. 27 (1981) 690-693.

[14] T.N.L. Vu, M. Lee, Independent design of multi-loop PI/PID controllers for interacting multivariable processes, J. Process Control 20 (2010) $922-933$. 
[15] Q. Xiong, W.J. Cai, Effective transfer function method for decentralized control system design of multi-input multi-output processes, J. Process Control 16 (2006) $773-784$.

[16] Q. Xiong, W.J. Cai, M.J. He, A practical loop pairing criterion for multivariable processes, J. Process Control 15 (2005) $741-747$.

[17] Y.L. Shen, W.J. Cai, S.Y. Li, Multivariable process control: decentralized, decoupling, or sparse? Ind. Eng. Chem. Res. 49 (2010) $761-771$.

[18] M.J. He, W.J. Cai, W. Ni, L.H. Xie, RNGA based control system configuration for multivariable processes, J. Process Control 19 (2009) $1036-1042$.

[19] Q.F. Liao, W.J. Cai, Y.Y. Wang, Effective T-S fuzzy model for decentralized control, in: 7th International Conference on Information and Automation for Sustainability: Sharpening the Future with Sustainable Technology, Colombo, Sri Lanka, 2014, pp. 1-6.

[20] L.A. Zadeh, The concept of a linguistic variable and its application to approximate reasoning-I, Inf. Sci. 8 (1975) $199-249$.

[21] N.N. Karnik, J.M. Mendel, L. Qilian, Type-2 fuzzy logic systems, IEEE Trans. Fuzzy Syst. 7 (1999) 643-658.

[22] J.M. Mendel, R.I.B. John, Type-2 fuzzy sets made simple, IEEE Trans. Fuzzy Syst. 10 (2002) 117-127.

[23] Q.L. Liang, J.M. Mendel, Interval type-2 fuzzy logic systems: theory and design, IEEE Trans. Fuzzy Syst. 8 (2000) $535-550$.

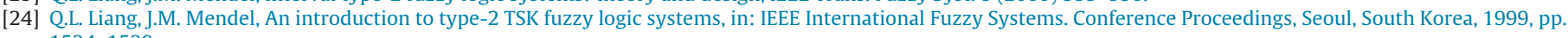
$1534-1539$.

[25] Q.F. Liao, N. Li, S.Y. Li, Type-II T-S fuzzy model-based predictive control, in: Proceedings of the IEEE Conference on Decision and Control, Shanghai, China, 2009, pp. 4193-4198.

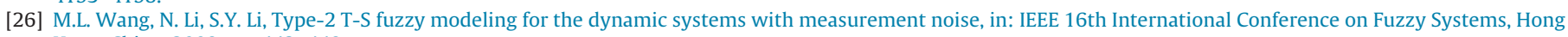
Kong, China, 2008, pp. 443-448.

[27] Q. Ren, L. Baron, M. Balazinski, Type-2 Takagi-Sugeno-Kang fuzzy logic modeling using subtractive clustering, in: Annual Meeting of the North American Fuzzy Information Processing Society, Montreal, Canada, 2006, pp. 120-125.

[28] Q.F. Liao, W.J. Cai, S.Y. Li, Y.Y. Wang, Interaction analysis and loop pairing for MIMO processes described by T-S fuzzy models, Fuzzy Sets Syst. 207 (2012) 64-76.

[29] L. Ljung, System Identification: Theory for the User, second ed., Prentice-Hall Inc., Englewood Cliffs, NJ, 1999.

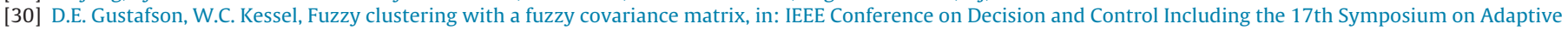
Processes, San Diego, USA, 1978, pp. 761-766.

[31] A. Niederlinski, A heuristic approach to the design of linear multivariable interacting subsystems, Automatica 7 (1971) $691-701$. 